\title{
Article \\ Genome-Wide Comprehensive Analysis of the GASA Gene Family in Populus
}

\author{
Shuo Han ${ }^{1,+}{ }^{+}$, Zhiyin Jiao ${ }^{1,+}$, Meng-Xue Niu ${ }^{1}$, Xiao Yu ${ }^{1}$, Mengbo Huang ${ }^{1}$, Chao Liu ${ }^{1}{ }^{1}$, Hou-Ling Wang ${ }^{1}{ }^{\circledR}$, \\ Yangyan Zhou ${ }^{2}$, Wei Mao ${ }^{2}$, Xiaofei Wang ${ }^{2}$, Weilun Yin ${ }^{1, *}$ and Xinli Xia ${ }^{1, *(D)}$ \\ 1 National Engineering Laboratory for Tree Breeding, College of Biological Sciences and Technology, Beijing \\ Forestry University, Beijing 100083, China; hanhan@bjfu.edu.cn (S.H.); jiaozy@bjfu.edu.cn (Z.J.); \\ niumengxue@bjfu.edu.cn (M.-X.N.); yuxiao@bjfu.edu.cn (X.Y.); hmb425069@bjfu.edu.cn (M.H.); \\ liuchao1306@bjfu.edu.cn (C.L.); whling@bjfu.edu.cn (H.-L.W.) \\ 2 Salver Academy of Botany, Rizhao 276800, China; zhou@salver.cn (Y.Z.); mao@salong-agriculture.com (W.M.); \\ wang@dfy.net.cn (X.W.) \\ * Correspondence: yinw1@bjfu.edu.cn (W.Y.); xiaxl@bjfu.edu.cn (X.X.); Tel.: +86-10-62336400 (X.X.) \\ + These authors contributed equally to this work.
}

check for updates

Citation: Han, S.; Jiao, Z.; Niu, M.-X.; Yu, X.; Huang, M.; Liu, C.;

Wang, H.-L.; Zhou, Y.; Mao, W.;

Wang, X.; et al. Genome-Wide

Comprehensive Analysis of the GASA

Gene Family in Populus. Int. J. Mol.

Sci. 2021, 22, 12336. https://doi.org/

10.3390/ijms222212336

Academic Editors:

Prabhakaran Soundararajan

and Abinaya Manivannan

Received: 17 September 2021

Accepted: 8 November 2021

Published: 15 November 2021

Publisher's Note: MDPI stays neutral with regard to jurisdictional claims in published maps and institutional affiliations.

Copyright: (c) 2021 by the authors. Licensee MDPI, Basel, Switzerland. This article is an open access article distributed under the terms and conditions of the Creative Commons Attribution (CC BY) license (https:// creativecommons.org/licenses/by/ $4.0 /)$.

\begin{abstract}
Gibberellic acid-stimulated Arabidopsis (GASA) proteins, as cysteine-rich peptides (CRPs), play roles in development and reproduction and biotic and abiotic stresses. Although the GASA gene family has been identified in plants, the knowledge about GASAs in Populus euphratica, the woody model plant for studying abiotic stress, remains limited. Here, we referenced the well-sequenced Populus trichocarpa genome, and identified the GASAs in the whole genome of P. euphratica and $P$. trichocarpa. 21 candidate genes in P. trichocarpa and 19 candidate genes in P. euphratica were identified and categorized into three subfamilies by phylogenetic analysis. Most GASAs with signal peptides were located extracellularly. The GASA genes in Populus have experienced multiple gene duplication events, especially in the subfamily A. The evolution of the subfamily A, with the largest number of members, can be attributed to whole-genome duplication (WGD) and tandem duplication (TD). Collinearity analysis showed that WGD genes played a leading role in the evolution of GASA genes subfamily B. The expression patterns of $P$. trichocarpa and P. euphratica were investigated using the PlantGenIE database and the real-time quantitative PCR (qRT-PCR), respectively. GASA genes in $P$. trichocarpa and P. euphratica were mainly expressed in young tissues and organs, and almost rarely expressed in mature leaves. GASA genes in P. euphratica leaves were also widely involved in hormone responses and drought stress responses. GUS activity assay showed that PeuGASA15 was widely present in various organs of the plant, especially in vascular bundles, and was induced by auxin and inhibited by mannitol dramatically. In summary, this present study provides a theoretical foundation for further research on the function of GASA genes in P. euphratica.
\end{abstract}

Keywords: GASA genes; bioinformatics; Populus; growth and development; phytohormone

\section{Introduction}

Cysteine-rich peptides (CRPs), with a secretory peptide signal at the N-terminal region and a cysteine-rich domain containing 4-16 cysteine residues at the C-terminal region, are generally small (usually less than 160 amino acid residues) and have been increasingly studied in plant [1-4]. CRPs, as plant peptide ligands that trigger membrane receptors, play a vital role in plant development and reproduction, plant-bacteria symbiosis, and plant defense, and are widely recognized as important signaling components that coordinate cell-cell communication in plants [1,5-7]. Gibberellic acid-stimulated Arabidopsis (GASA) proteins are classified as cysteine-rich peptides consisting of a signal peptide at the N-terminal, a highly variable hydrophilic region in the middle, and a conserved region containing 12 cysteine residues at the C-terminal (GASA domain) [8]. GASA proteins are 
widely found in vascular terrestrial plants such as pteridophytes, gymnosperms, and angiosperms, which have significance for adapting to complex and diverse land life [9]. Since gibberellin-stimulated transcript 1 (GAST1) was first identified in tomato GA-deficient gib1 mutant, an increasing number of GASA members have been identified over the past three decades [10].

Snakin-1 (SN1) and snakin-2 (SN2), which were classified as members of the GASA family, have antibacterial activity. Overexpression of SN1 in potatoes and SN2 in tomatoes inhibited the invasion of pathogenic bacteria and enhanced the tolerance to bacterial and fungal presence [11,12]. Likewise, the allogenic expression of StSN1 could improve the tolerance to fungal pathogens in transgenic lettuce plants [13]. PdSN1, the homologous protein of potato snakin-1 in Peltophorum dubium, inhibited the growth of multiple plants and animal pathogens in vitro [14]. GASA proteins also participate in plant growth and development [15-19]. The silencing of SN1 in potatoes affected cell division, primary metabolism and cell wall composition of transgenic plants, eventually leading to changes in leaf size and shape and plant dwarfing [20]. AtGASA5 delayed flowering time by enhancing the expression of $F L C$ and repressing the expression of FT and LEAFY [17]. GhGEG, a gene from the GASA gene family in Gerbera hybrida, was directly transcriptional activated by GhMIF and GhEIL1 to inhibit the ray petal elongation and cell elongation during petal development [21,22]. AtGASA4 regulated the differentiation and development of flower development meristem and increases the seed size and yield of Arabidopsis. [23]. FaGAST2 and FaGAST1 are involved in regulating the size of strawberry fruits, during the development and ripening of strawberry fruits [16]. AtGASA10 is strongly expressed in developing anthers, seeds, and the vasculature of elongating roots, and overexpression of AtGASA10 in transgenic Arabidopsis might reduce the length of the silique by affecting cell elongation [24]. And ZmGSL1, ZmGSL2, ZmGSL4, ZmGSL6, and ZmGSL9, GASAs in Zea mays, contributed to lateral root formation and development in maize [19,25].

GASAs are also involved in the transduction of hormone signals [26]. OsGSR1 in the rice GASA gene family, a positive regulator of GA signaling, interacted with DIM/DWF1 to regulate BR synthesis, and mediated the interaction between BR and GA signaling pathways [8]. As an integrator of gibberellin, abscisic acid and glucose signaling, AtGASA6 connected the functions of RGL2 and AtEXPA1, and regulated seed germination and hypocotyl length [18]. The expression of GASA family genes was responsive to exogenous treatment of growth hormones and stress hormones [15,21,27-29]. Exogenous $\mathrm{GA}_{3}$ inhibited the expression of most MdGASA genes in apple flowers, except the AtGASA10 homologous gene MdGASA1/6/7/19, HbGASA7-1, HbGASA14-3, HbGASA15 were induced by stress hormones salicylic acid, abscisic acid, ethylene and methyl jasmonate in Hevea brasiliensis [27]. The GASA genes participate in the regulation of plant strategies under stress. For example, transcriptomic and qRT-PCR analyses revealed the expression of GASAs was induced or inhibited by abiotic stresses such as cold, heat, drought, osmotic, salt, and wounding [25,30,31]. Overexpression of AtGASA14 reduced the accumulation of reactive oxygen species (ROS) to resist ABA and high salt stress in transgenic Arabidopsis [32]. The allogenic expression of SmGASA4 was found to promote the development of Arabidopsis roots and enhance plant resistance to salt and drought stress [31].

Plants are frequently subjected to various environmental stresses, especially abiotic stresses, which inhibit plant growth and biomass accumulation. P. euphratica, which can survive in extremely arid desert ecosystems, is widely regarded as an important model plant for studying woody plants in response to abiotic stresses [33-36]. In our previous studies, two GASA genes were abundant in transcripts, one of which was homologous to AtGASA1 and significantly up-regulated in drought-stressed P. euphratica leaves [37,38]. However, GASA1 was down-regulated by drought in Arabidopsis [39]. Although they are homologous genes from different plants, they have different expression patterns in response to drought stress. It is necessary to identify the GASA gene family and research the functions of the GASA gene family in the growth and development and stress response of P. euphratica. However, little information concerning GASA genes in P. euphratica has 
been known. Recently, the genome of $P$. euphratica has been sequenced and assembled, making it possible to study the GASA gene family on a genome-wide scale $[36,40]$. While the full genome of P. euphratica v1.0 had not yet been assembled into chromosomes, and there were numerous gaps in the scaffolds $[40,41]$. The GASA proteins we searched in $P$. euphratica v2.0 were less than P. euphratica v1.0.

The genome of $P$. trichocarpa (black cottonwood) was well-sequenced and annotated and had high similarity and collinearity with that of P. euphratica. To ensure the reliability of the results, the GASA gene family was identified in the genome-wide of $P$. euphratica by referring to the GASA gene family of $P$. trichocarpa [42]. In this study, we identified 21 PtrGASA genes and 19 PeuGASA candidate genes. Chromosomal localization, gene expansion, gene structure, and upstream promoter cis-acting elements of candidate GASA genes were predicted and analyzed. The chemical properties, subcellular localization, motifs, and phylogenetic relationships of the proteins encoded by them were also predicted and analyzed. Furthermore, the expression patterns of these genes in different organs and tissues and their responses to drought stress and exogenous hormones were investigated. The temporal and spatial expression patterns of the representative gene PeuGASA15 and its response to hormones and stresses were analyzed. This study represents the first comprehensive analysis of GASA genes in P. euphratica and P. trichocarpa and provided a theoretical basis for further study on the structures and functions of Populus GASA genes. Our findings might serve as a valuable resource for future studies of GASA genes related to vascular tissue development in P. euphratica as well as in other woody plants.

\section{Results}

\subsection{Genome-Wide Identification of GASA Genes in Populus}

To identify the members of the GASA gene family in P. euphratica, we searched for the conserved GASA domain (PF02704) in the whole genome protein database reported by Ma et al. and Zhang et al. [36,40]. We found 19 candidate GASA protein sequences in the protein database reported by Ma et al., two of which were encoded by the same gene LOC105128593 in different splicing ways (Table 1a). Fourteen GASA protein sequences were found in the protein database reported by Zhang et al., and 12 of which were identical to those reported by Ma et al. (Table 1b). GWHGAAYU001024 is homologous to AtGASA14 and encodes 158 amino acids. However, the same gene LOC105141680 encodes 222 amino acids as reported by Ma et al., which may be due to different splicing patterns in the intermediate hydrophilic region. The protein sequence of GWHPAAYU001570 was $98.86 \%$ identical with the homologous protein sequence in P. trichocarpa. Combined with two protein databases, we identified 19 GASA proteins in P. euphratica and named them according to gene ID on NCBI (Supplementary Table S1). 21 GASA genes with conserved GASA domains were identified in P. trichocarpa on Popgenie and named according to their position on chromosomes (Supplementary Table S1).

Except for the fact that PtrGASA21 was not assembled on any chromosome, $\operatorname{PtrGASA}$ genes were distributed on 12 chromosomes in $P$. trichocarpa genome (Figure 1). Four PtrGASA genes were found on chromosome 1, three PtrGASA genes were found on chromosomes 2, two PtrGASA genes were found on chromosomes 5, 7, and 17 respectively, and only one $\operatorname{Pt} G A S A$ gene was found on the remaining seven chromosomes. According to the positions of homologous $\operatorname{PtrGASAs}$ or linked genes, the possible chromosomal position of PeuGASAs was determined (Supplementary Table S1 and Figure 1). Almost all PeuGASAs had a corresponding position on the $P$. trichocarpa chromosomes except for two pairs of PeuGASA genes (PeuGASA1 and PeuGASA2; PeuGASA3 and PeuGASA13). Three PeuGASA genes were found on chromosomes 1, 2, and 5 of $P$. trichocarpa, which were the chromosomes containing the most PeuGASA genes. PtrGASA duplicate gene clusters were found on chromosomes 2, 5, and 7 in P. trichocarpa, while no corresponding PeuGASA homologous gene or PeuGASA duplicate gene cluster was found on chromosome 7. 
Table 1. Identification of GASA gene family in P. euphratica.

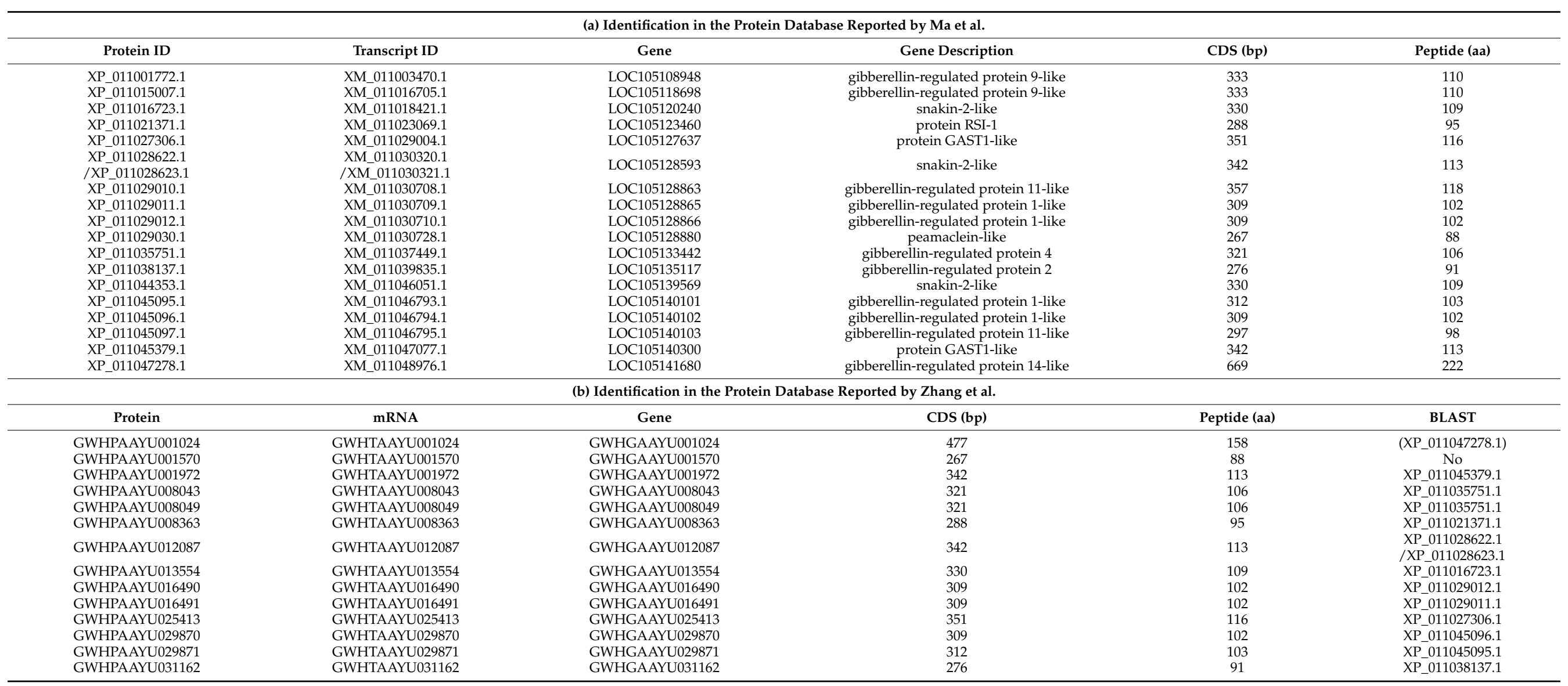




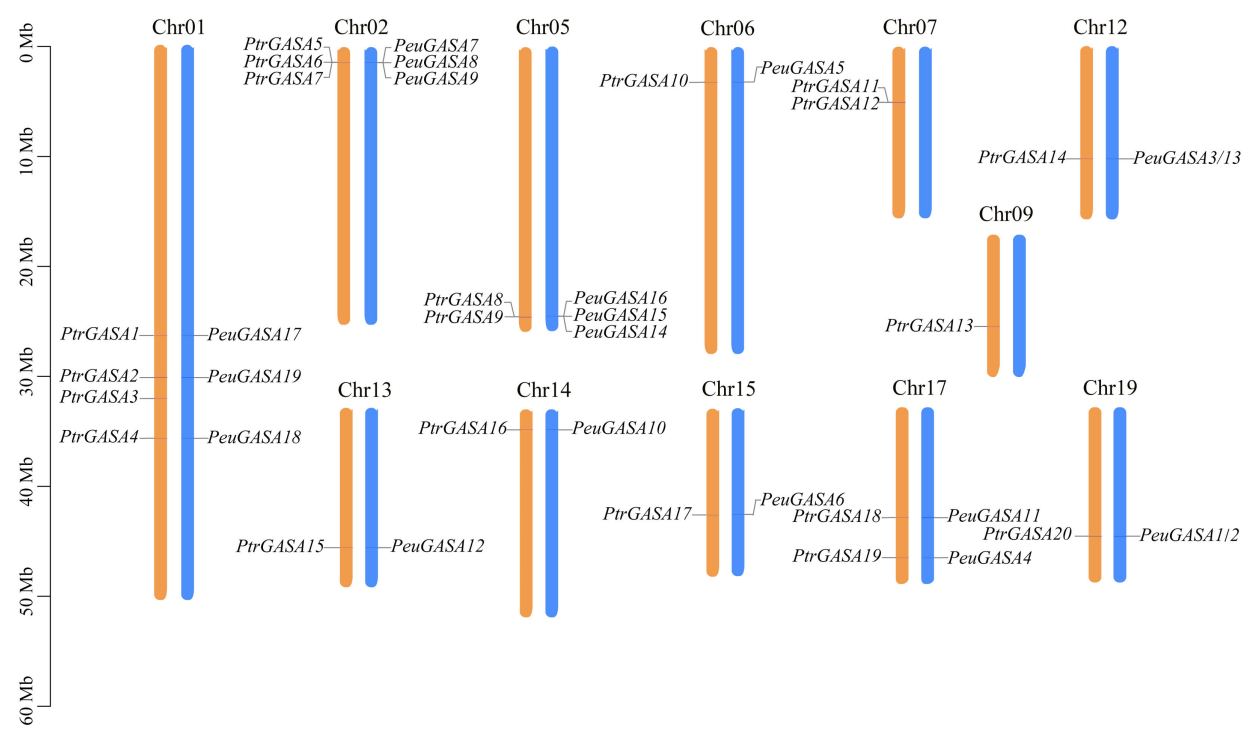

Figure 1. Positions of PtrGASA and PeuGASA genes on P. trichocarpa chromosomes. The P. trichocarpa chromosomes where the PtrGASA genes are located are shown in orange. The reference $P$. trichocarpa chromosomes where the PeuGASA genes are located are shown in blue. The positions of genes and the size of chromosomes can be estimated using the scale on the left of the picture.

2.2. Evolutionary Relationships Analysis among GASA Domains and Collinearity Analysis among GASA Genes

To illustrate the evolutionary relationship of the GASA gene family, the phylogenetic tree of the conserved GASA domains was constructed. Except for the GASA domains of $S$. moellendorffii and P. abies, which were separated into a distal branch, the remaining GASA domains were divided into three subfamilies: subfamily A (green), subfamily B (red), subfamily C (blue) (Figure 2). Compared with the other two subfamilies, subfamily A had more members and higher rates of substitution. The GASA domain of S. moellendorffii was not found in the subfamily A. MA_9071020g0010, MA_40654g0010, MA_9084305g0010, MA_717315g0010, MA_104045g0010, MA_153079g0010, and MA_307539g0010, with low substitution rates, clustered together and diverged from PtrGASA20, PeuGASA1, PeuGASA2, and AtGASA9. While PtrGASA4, PeuGASA18, PtrGASA17, PeuGASA6, PtrGASA14, PeuGASA13, and PeuGASA3, with high substitution rates, clustered together and separated hierarchically from OsGASR4, MA 23745g0010, and AtGASA14 which evolved earlier. In subfamily B, the GASA domains of S. moellendorffii, P. abies, O. sativa, A. thaliana, and Populus each had a successive independent clade within the species. OsGASR8 and SM00051G00360, with similar sequences and high substitution rates, clustered together and diverged from SM00013G01380. OsGASR1 and SM00035G00570, with relatively low substitution rates, separated earlier in the subfamily $C$ hierarchically. In the subfamily C, except for a pair of GASA domains of P. abies and the homologous GASA domains of Populus, the GASA domains dispersed on several small branches had low substitution rates. Compared with GASA domains of other species, GASA domains of Arabidopsis and Populus had higher substitution rates and homology. 


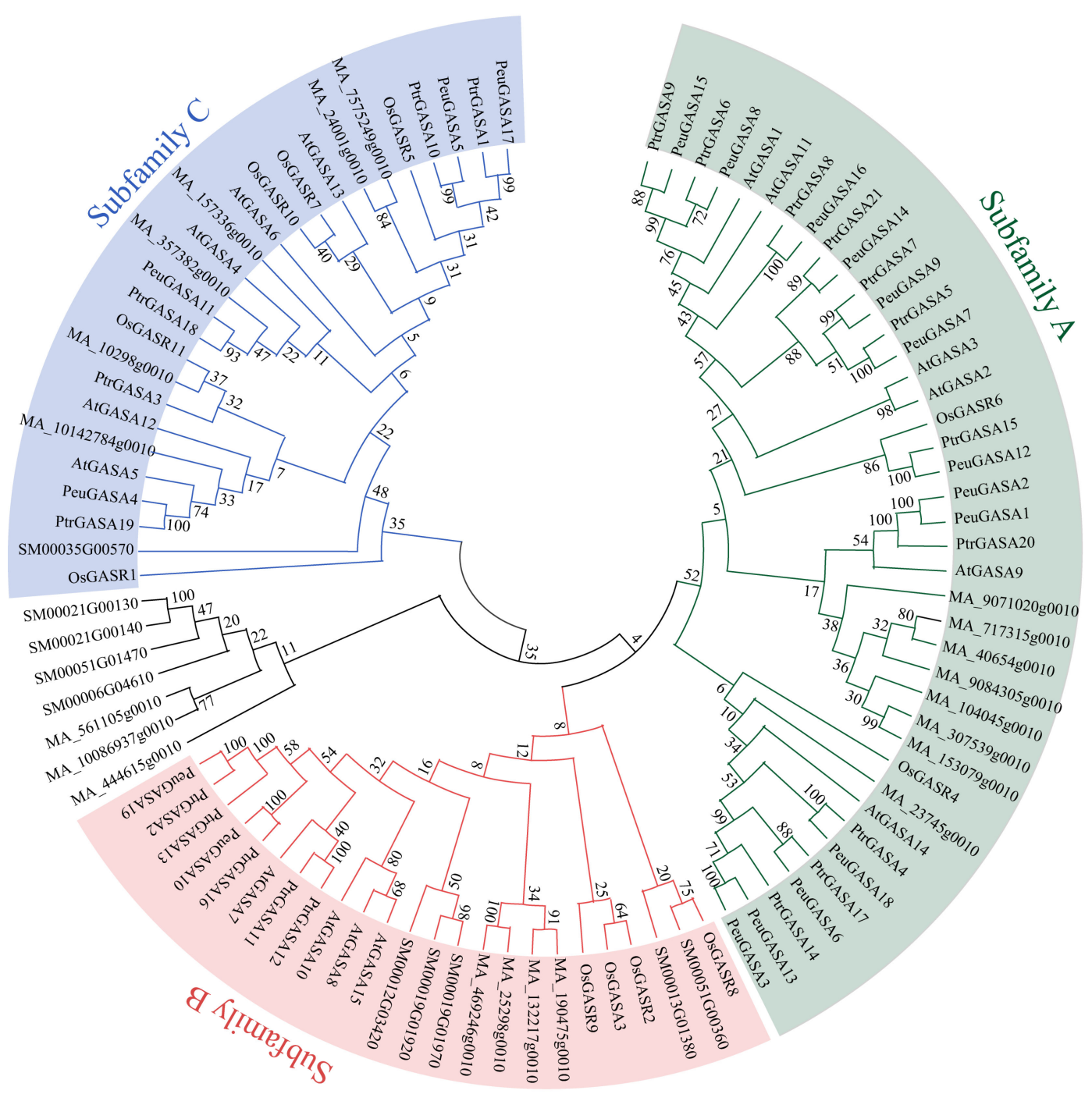

Figure 2. Neighbor-Joining phylogenetic tree of GASA domains. The 97 conserved GASA domains of GASAs in sequenced plants, Selaginella moellendorffii, Picea abies, Oryza sativa ssp. japonica, Arabidopsis thaliana, Populus trichocarpa, and Populous euphratica were analyzed. The tree was divided into three phylogenetic subgroups, which were shown in different colors.

To illustrate the expansion pattern of GASA genes, we analyzed the duplicate events in the genomes of P. trichocarpa and P. euphratica (Table 2). Multiple duplicate events occurred in the evolution of PtrGASAs and PeuGASAs. Each pair in PtrGASA2, PtrGASA11, PtrGASA13, and PtrGASA16 was a WGD pair, indicating that they all share a common ancestor. The WGD pairs and TD pairs of the PeuGASAs and PtrGASAs homologous genes were almost identical. The lack of duplicate genes in PtrGASAs and PeuGASAs, which may be due to genes loss caused by the chromosome dynamic rearrangement after whole-genome duplications. The $\mathrm{Ka} / \mathrm{Ks}$ values of each duplicated pair were much less than 1 , indicating that these genes were selected for purification. The $K a / K s$ values of the duplicated pairs in P. euphratica were slightly higher than those in P. trichocarpa, indicating that GASA genes in P. trichocarpa might be more conserved. Unlike the multiple pairs of duplications in poplar, only a WGD pair and a TD pair were found in Arabidopsis (AtGASA3 and AtGASA9, AtGASA2 and AtGASA3). Collinear relationships of GASAs in poplar and Arabidopsis showed that 21 pairs of homologous genes were found in $P$. trichocarpa and Arabidopsis, which were composed of 7 AtGASA genes and 15 PtrGASA genes. There were 12 pairs of homologous genes between P. trichocarpa and Arabidopsis (Figure 3). 


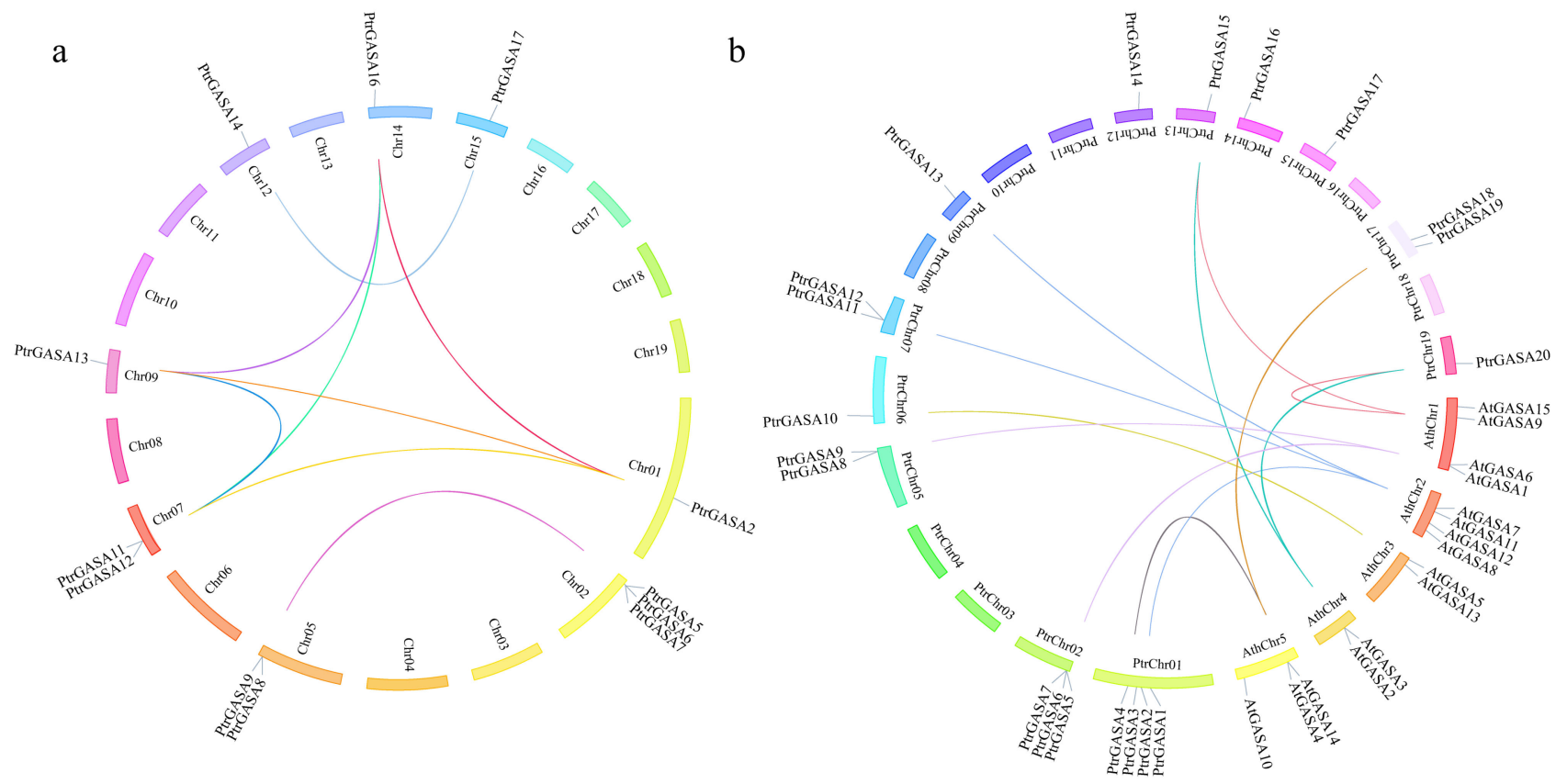

Figure 3. Analysis of the evolutionary relationship among GASA genes. (a) Evolution analysis of GASA gene family in $P$. trichocarpa; (b) Collinear analysis of GASA gene family between the Arabidopsis and P. trichocarpa. Different sizes of fan rings represented different sizes of chromosomes. The colored lines represented collinear GASA gene pairs.

Table 2. Duplications of GASA genes in P. euphratica.

\begin{tabular}{|c|c|c|c|c|c|c|c|}
\hline Gene 1 & Gene 2 & Duplication & E-Value & $K a$ & $K s$ & $K a / K s$ & Selection Pressure \\
\hline PtrGASA2 & PtrGASA11 & WGD & $5 E-16$ & 0.306574081 & 1.511177265 & 0.202871025 & Purifying selection \\
\hline PtrGASA2 & PtrGASA13 & WGD & $1 \mathrm{E}-41$ & 0.024592367 & 0.245409683 & 0.100209441 & Purifying selection \\
\hline PtrGASA2 & PtrGASA16 & WGD & $3 E-34$ & 0.189006113 & 0.854755332 & 0.221123058 & Purifying selection \\
\hline PtrGASA11 & PtrGASA13 & WGD & $8 \mathrm{E}-14$ & 0.314370832 & 1.291132145 & 0.24348463 & Purifying selection \\
\hline PtrGASA11 & PtrGASA16 & WGD & $2 \mathrm{E}-14$ & 0.2812637 & 1.166421628 & 0.241133818 & Purifying selection \\
\hline PtrGASAd13 & PtrGASA16 & WGD & $6 \mathrm{E}-34$ & 0.175785289 & 1.055787598 & 0.166496831 & Purifying selection \\
\hline PtrGASA7 & PtrGASA9 & WGD & $1 \mathrm{E}-33$ & 0.189204442 & 0.883649795 & 0.214116998 & Purifying selection \\
\hline PtrGASA14 & PtrGASA17 & WGD & $4 \mathrm{E}-68$ & 0.041106177 & 0.225565616 & 0.182236008 & Purifying selection \\
\hline PtrGASA5 & PtrGASA6 & TD & $4 \mathrm{E}-31$ & 0.257142422 & 0.939998969 & 0.273556068 & Purifying selection \\
\hline PtrGASA6 & PtrGASA7 & TD & $1 \mathrm{E}-34$ & 0.162561762 & 0.966817469 & 0.16814111 & Purifying selection \\
\hline PtrGASA8 & PtrGASA9 & TD & $7 \mathrm{E}-34$ & 0.320382084 & 1.907862372 & 0.167927251 & Purifying selection \\
\hline PtrGASA11 & PtrGASA12 & TD & $4 \mathrm{E}-30$ & 0 & 0 & $\mathrm{NaN}$ & - \\
\hline PeuGASA6 & PeuGASA13 & WGD & $6 \mathrm{E}-49$ & 0.057332466 & 0.184523724 & 0.31070512 & Purifying selection \\
\hline PeuGASA9 & PeuGASA15 & WGD & $2 \mathrm{E}-39$ & 0.097292607 & 0.362138829 & 0.268661075 & Purifying selection \\
\hline PeuGASA10 & PeuGASA19 & WGD & $2 \mathrm{E}-34$ & 0.195458562 & 0.965443142 & 0.202454762 & Purifying selection \\
\hline PeuGASA7 & PeuGASA8 & TD & $2 \mathrm{E}-32$ & 0.204373366 & 0.898115959 & 0.227557883 & Purifying selection \\
\hline PeuGASA8 & PeuGASA9 & TD & $6 \mathrm{E}-30$ & 0.185223217 & 0.962509794 & 0.192437747 & Purifying selection \\
\hline PeuGASA14 & PeuGASA15 & TD & $1 \mathrm{E}-44$ & 0.253240964 & 0.898681597 & 0.281791644 & Purifying selection \\
\hline PeuGASA15 & PeuGASA16 & TD & $3 E-35$ & 0.297194132 & 1.782104232 & 0.166765853 & Purifying selection \\
\hline
\end{tabular}

2.3. Gene Structure and Motif Identification, Physicochemical Properties, and Subcellular Localization of GASA Gene Family

To illustrate the diversity of GASA genes in poplar, the arrangement of introns-exons was compared according to their phylogenetic relationships (Figure 4). As shown in Figure $4 \mathrm{~b}$, genes in subfamily B were composed of two exons and one intron. Except for PtrGASA3, GASA genes which were present in $P$. trichocarpa but had no corresponding homologous genes in $P$. euphratica belong to this subfamily. The members of the GASA gene family contain no more than three introns. The predicted motifs of GASA proteins were mainly concentrated at the C-terminal of GASA proteins. GASA domain was jointly constituted by motif 1 , motif 2, motif 4, and motif 5 . Motif 3 , which was located at the N-terminal of the proteins and contained a large amount of the hydrophobic amino acid leucine. The multiple sequence alignment identity of 40 GASA proteins was only $26.70 \%$, while that of 40 GASA domains was $66 \%$. The conserved domain of GASA was composed of 59 or 60 amino acids, including 12 cysteine residues with 
conserved positions (Supplementary Figure S1a). Despite the lack of motif 2 and low similarity with other subfamily amino acid sequences, GASA proteins in subfamily B had conserved GASA domains.

a

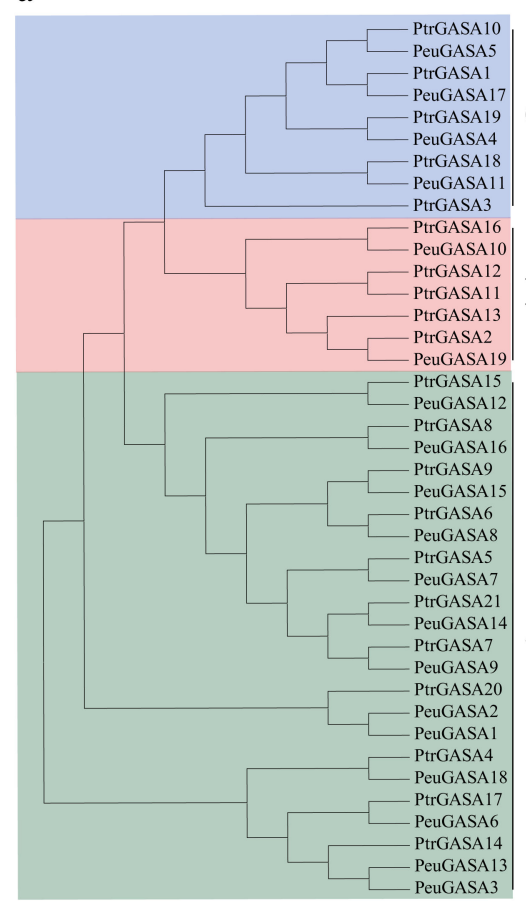

$\mathrm{b}$

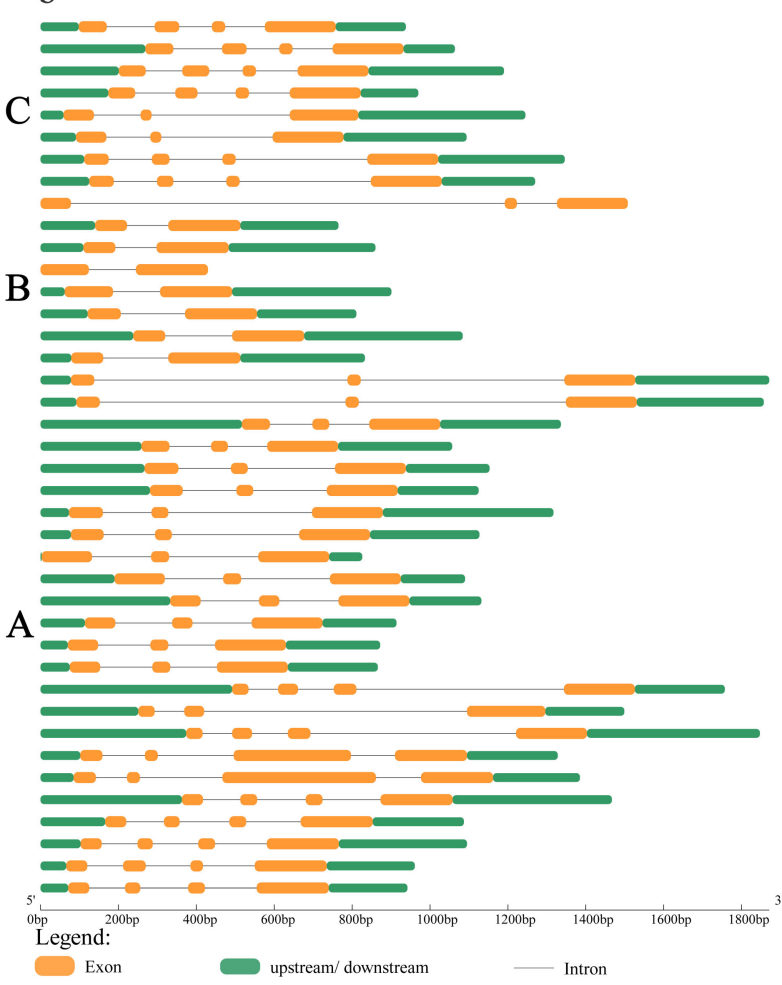

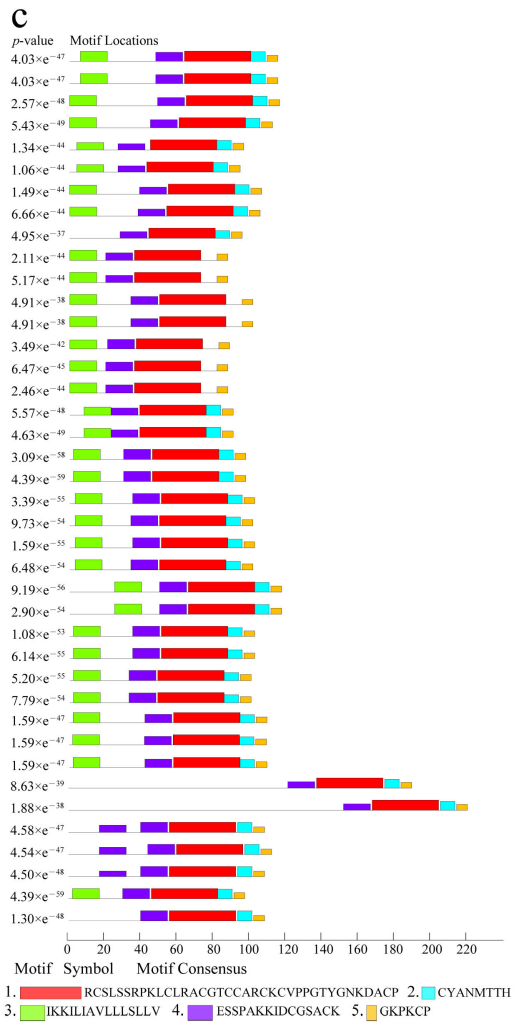

Figure 4. Gene structures and motif analysis of GASA gene family in P. euphratica and P. trichocarpa. (a) Phylogenetic tree of PtrGASAs and PeuGASAs. Subfamily A/B/C were represented by green, red and blue respectively; (b) Gene structures of PtrGASAs and PeuGASAs. Upstream or downstream non-coding regions were represented by green rounded rectangles, exons by orange rounded rectangles, and introns by gray lines; (c) Conserved motifs of PtrGASAs and PeuGASAs. Conservative motifs were presented in different colored boxes. The length of each nucleotide sequence or protein sequence can be estimated using the scale below the picture.

GASA genes in Populus encoded 88-118 amino acids, except for AtGASA14 homologous genes PeuGASA18 and PtrGASA4, which encoded about 200 amino acids. The molecular weight of GASA proteins was relatively small, almost less than $13 \mathrm{kDa}$, except for PeuGASA18 and PtrGASA4. The isoelectric point of GASA proteins was ranged from 7.96 to 9.76 , which belonged to basic protein. The GASAs in Populus were mainly unstable proteins and signal peptides secreted through the classical secretion pathway (Supplementary Tables S2 and S3). The predicted subcellular localization of GASA proteins in P. euphratica and $P$. trichocarpa mainly in extra (Supplementary Table S2). Consistent with previously predicted subcellular localization, the GASA proteins in P. euphratica were localized in the extracellular region (Figure 5). 


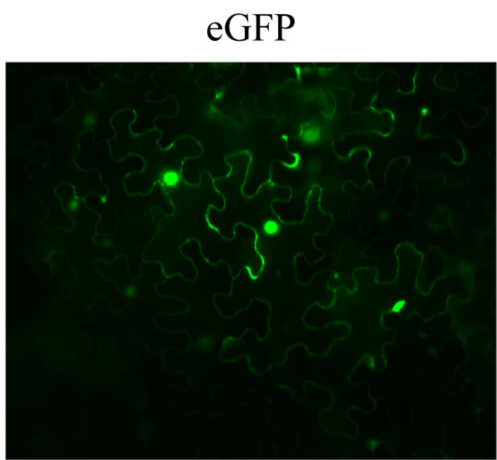

PeuGASA8-eGFP

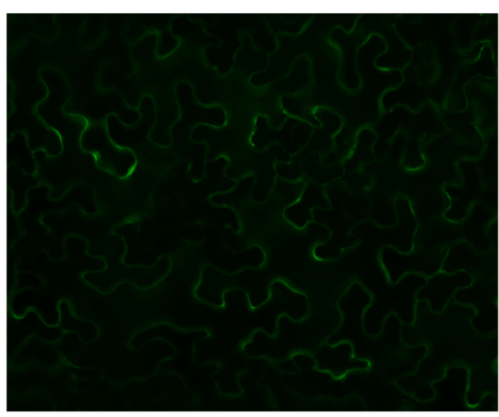

PeuGASA15-eGFP

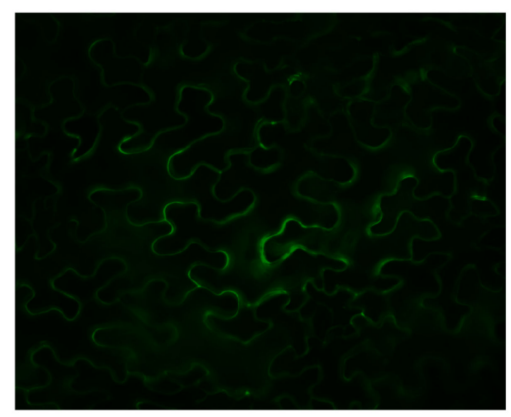

PeuGASA4-eGFP

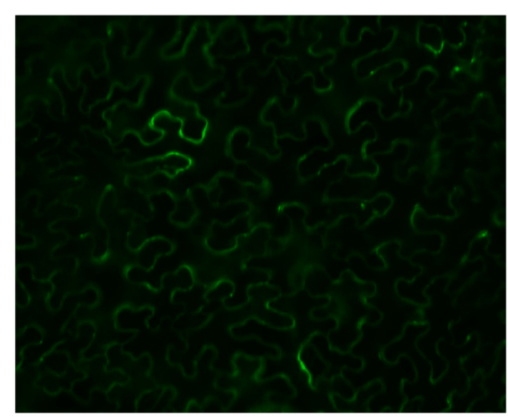

PeuGASA10-eGFP

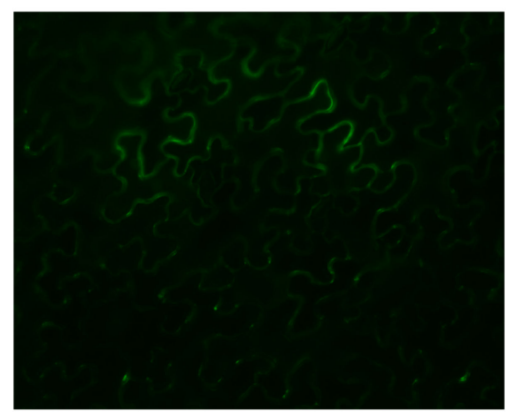

PeuGASA17-eGFP

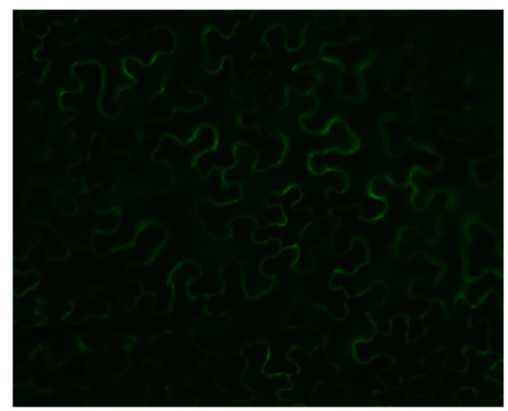

PeuGASA5-eGFP

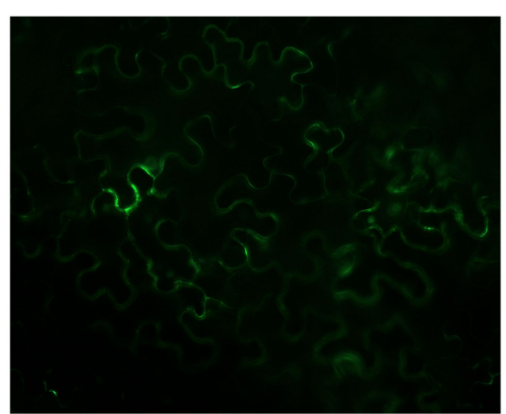

PeuGASA11-eGFP

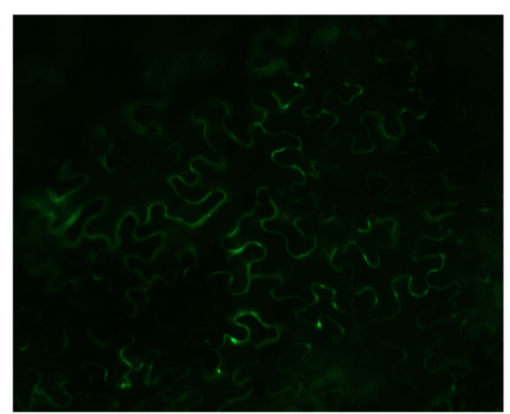

PeuGASA18-eGFP

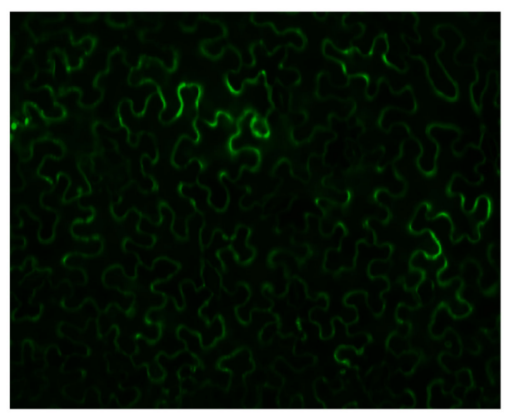

Figure 5. Subcellular localization of PeuGASA proteins. The Super::GFP and Super::PeuGASA-GFP fusion proteins were transiently expressed in tobacco.

\subsection{Expression Patterns of GASA Genes in Different Tissues of Populus}

The expression patterns of GASA genes in different tissues of $P$. trichocarpa were visualized in Supplementary Figure S2. PtrGASA12 was identified unexpressed in the selected dataset. The expression of PtrGASA3 and PtrGASA11 were hardly detected in $P$. trichocarpa. GASA genes in P. trichocarpa were highly expressed mainly in the stages of buds dormant, buds pre-chilling, leaves young expanding, leaves freshly expanded, flowers dormant and flowers expanding. And the expression levels decreased with leaf extension and flower opening. PtrGASA9, PtrGASA16, PtrGASA18, and PtrGASA21 were highly expressed in wood, PtrGASA18 and PtrGASA7 were highly expressed in root. Similar to the expression patterns of PtrGASAs, the expression levels of PeuGASA genes in young leaves were significantly higher than those in other tissues and organs, while the expression levels of PeuGASA genes in mature leaves were significantly lower than these in other tissues and organs (Figure 6). Compared with mature leaves, the expression levels of PeuGASA4, PeuGASA6, PeuGASA8, PeuGASA10, PeuGASA11, PeuGASA15, PeuGASA16, and PeuGASA17 were higher in old leaves. PeuGASAs were also expressed in the vascular tissues. PeuGASA1/2, PeuGASA6, PeuGASA11, PeuGASA14, and PeuGASA15 were highly expressed in phloem, and PeuGASA1/2, PeuGASA6, PeuGASA10, PeuGASA14, PeuGASA15, PeuGASA16, and PeuGASA19 were highly expressed in xylem. Except for PeuGASA8 and 
PeuGASA15, the expression levels of PeuGASAs in roots were not as high as that in young leaves, but mostly higher than that in mature leaves.
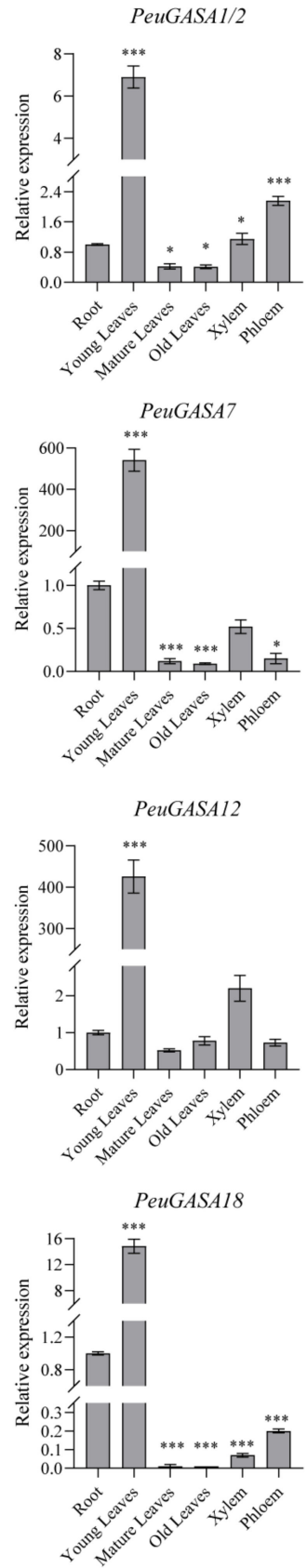

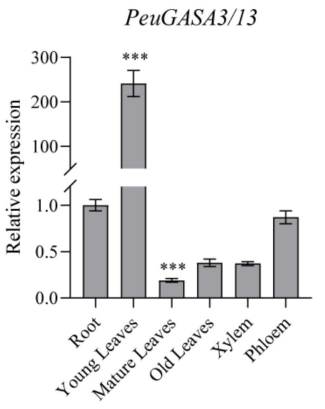

PeuGASA8

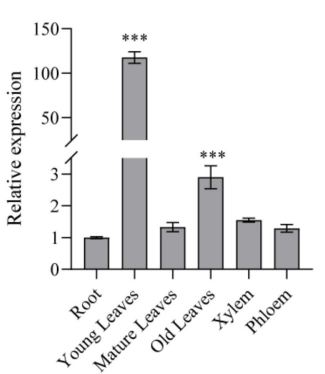

PeuGASA14
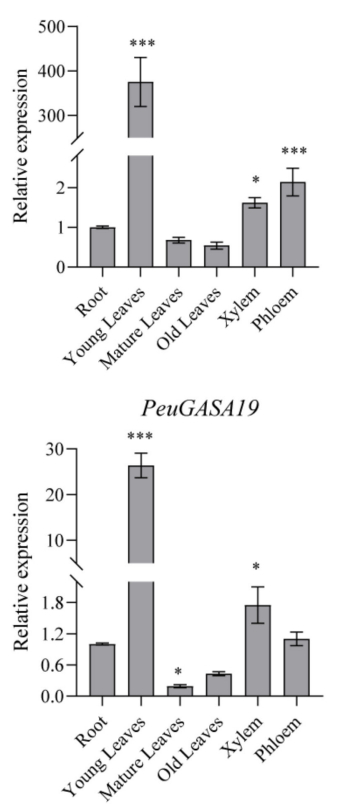

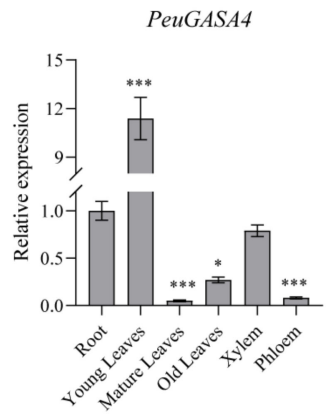

PeuGASA9
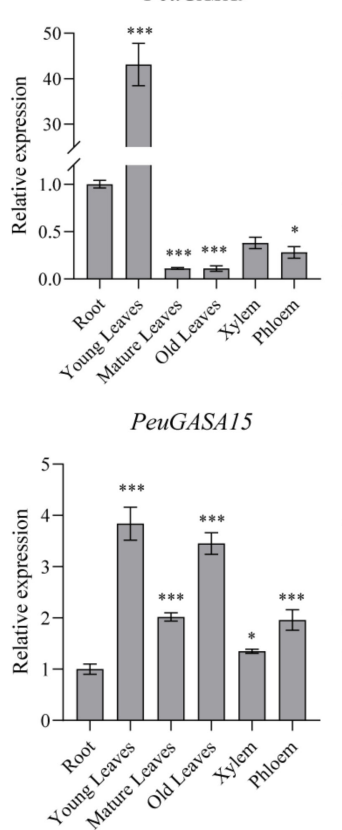

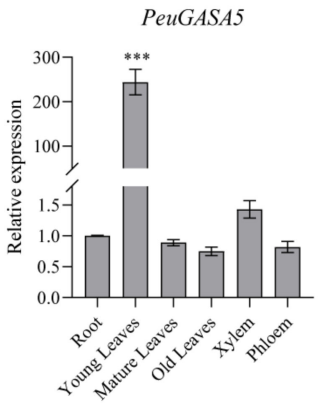

PeuGASA10
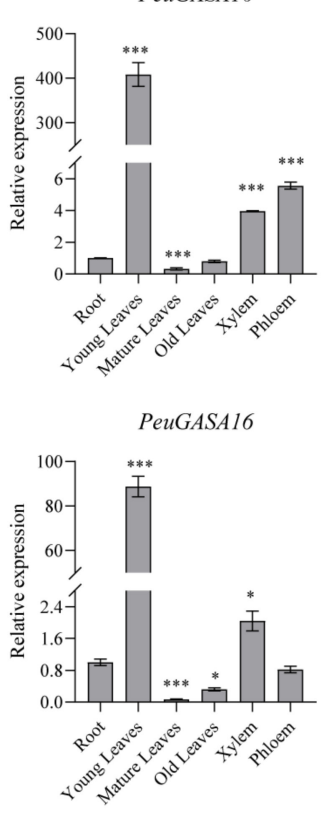

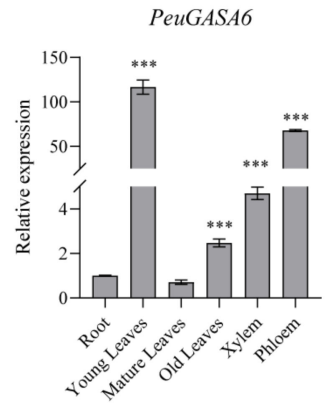

PeuGASA11
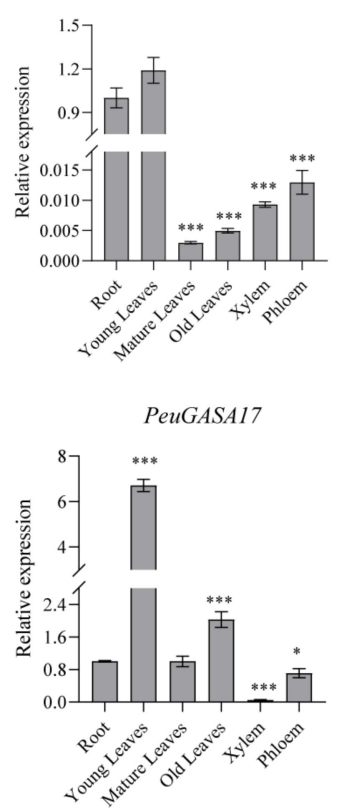

Figure 6. Expression patterns of GASA genes in different $P$. euphratica tissues. Data were presented as mean \pm standard error (SE). Subsequent multiple comparisons were evaluated based on the least significant difference (LSD) test to calculate $p$-values (Asterisks denoted significant differences: ${ }^{*} p \leq 0.05 ;{ }^{* * *} p \leq 0.01$ ).

\subsection{Responses of GASAs to Exogenous Hormones in P. euphratica Leaves}

To investigate the responses of GASA genes to exogenous hormones in P. euphratica leaves, the transcriptional levels of candidate genes were studied (Figure 7). After treatment with the stress hormone abscisic acid (ABA), the expression levels of PeuGASA8, PeuGASA11, PeuGASA15, PeuGASA17, and PeuGASA18 were down-regulated, the expression levels of $P e u G A S A 1 / 2$ and $P e u G A S A 16$ were initially down-regulated and up-regulated subsequently, but the expression levels of PeuGASA9, PeuGASA10 and PeuGASA14 were up-regulated. After being treated with salicylic acid (SA), the expression levels of Peu- 
GASA6, PeuGASA10, and PeuGASA14 were significantly up-regulated and the expression levels of PeuGASA5, PeuGASA7, PeuGASA11, and PeuGASA17 were significantly downregulated. The expression levels of PeuGASA1/2, PeuGASA4, PeuGASA8, PeuGASA9, and PeuGASA15 were down-regulated in the leaves of P. euphratica treated by methyl jasmonate (MeJA), while the expression levels of PeuGASA6, PeuGASA10, PeuGASA14, PeuGASA16, and PeuGASA17 were initially up-regulated and then down-regulated. After auxin (IAA) treatment, the expression levels of PeuGASA genes in P. euphratica leaves were sharply up-regulated, even increased dozens of times. The expression levels of PeuGASA1/2, PeuGASA5, PeuGASA6, PeuGASA9, PeuGASA12, PeuGASA14, and PeuGASA17 were upregulated in P. euphratica leaves after 24-epibrassinolide (EBR) treatment, while PeuGASA11 and PeuGASA15 were down-regulated. After gibberellin $\left(\mathrm{GA}_{3}\right)$ treated, the expressions PeuGASA1/2, PeuGASA4, PeuGASA8, PeuGASA9, PeuGASA10, PeuGASA11, PeuGASA14, and PeuGASA15 were inhibited, while the expressions of PeuGASA3/13, PeuGASA5, PeuGASA6, PeuGASA12, PeuGASA17, and PeuGASA19 were induced.

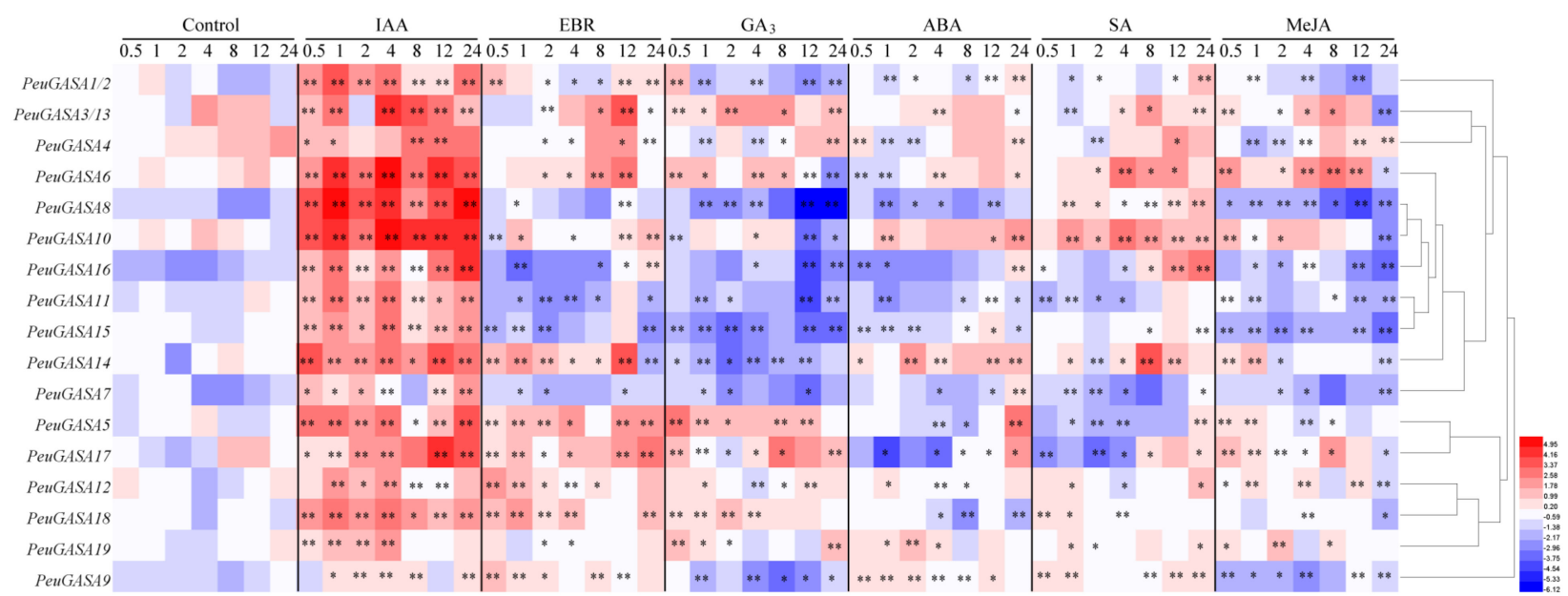

Figure 7. Expression analysis of PeuGASA genes in response to SA, ABA, MeJA, EBR, $\mathrm{GA}_{3}$ and IAA treatments. Numbers are the time $(\mathrm{h})$ of treatment. The heat map was based on the calculation of three replicates of qRT-PCR data and the transformation of $\log 2$ algorithm. Multiple comparisons were evaluated based on the least significant difference (LSD) test to calculate $p$-values (Asterisks denoted significant differences: ${ }^{*} p \leq 0.05$; ${ }^{* *} p \leq 0.01$ ).

\subsection{Response of GASAs to Stresses in Populus Leaves}

To further explore the function of GASA, GASA genes respond to stresses in Populus leaves had been investigated (Figure 8 and Supplementary Figure S3). No changes were detected in the expressions of PtrGASA3, PtrGASA5, PtrGASA11, and PtrGASA15 after drought, beetle damage, and mechanical damage (Supplementary Figure S3). No changes in the expression levels of PtrGASA7, PtrGASA13, and PtrGASA21 were detected after beetle damage and mechanical damage. After beetle damage and mechanical damage, the expressions of most $P \operatorname{trGASA}$ genes were strongly inhibited, while the expression of PtrGASA9 was strongly induced. Mechanical damage slightly induced the expression of $\operatorname{trGASA19}$. Another exception was that the expression of PtrGASA6 was inhibited by beetle damage but induced by mechanical damage. Compared with control group, the expressions of PtrGASA2, PtrGASA4, PtrGASA6, PtrGASA7, PtrGASA8, PtrGASA9, PtrGASA13, PtrGASA19, and PtrGASA21 were induced, while the expressions of PtrGASA1, PtrGASA10, PtrGASA14, PtrGASA16, PtrGASA17, PtrGASA18, and PtrGASA20 were inhibited in drought stress. Similar to $P$. trichocarpa, the expressions of GASA genes in $P$. euphratica leaves responded to drought stress. Drought stress induced the expression of PeuGASA1, PeuGASA2, PeuGASA4, PeuGASA15, and PeuGASA19, but inhibited the expression of PeuGASA3, PeuGASA5, PeuGASA6, PeuGASA8, PeuGASA10, PeuGASA11, PeuGASA12, PeuGASA13, PeuGASA14, PeuGASA16, and PeuGASA18 (Figure 8). 

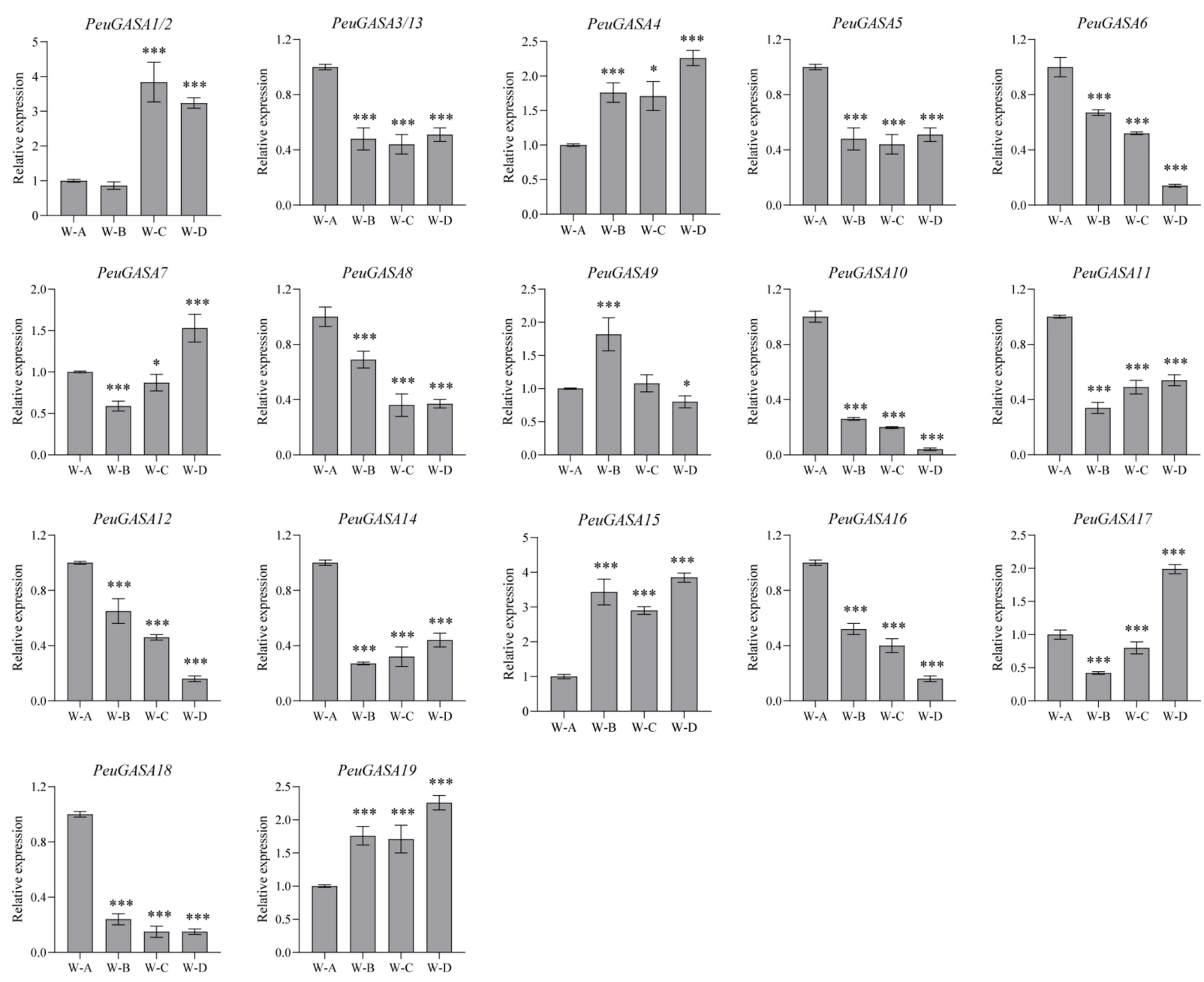

Figure 8. Expression analysis of PeuGASA genes in response to drought stress. W-A, W-B, W-C, and W-D respectively represented four drought treatments with different soil volumetric water content (soil-VWC): control (43 $\pm 1 \%$ soil-VWC), mild drought $(33 \pm 1 \%$ soil-VWC), moderate drought $(23 \pm 1 \%$ soil-VWC) and severe drought $(13 \pm 1 \%$ soil-VWC). Data were presented as mean \pm standard error (SE). Subsequent multiple comparisons were evaluated based on the least significant difference (LSD) test to calculate $p$-values (Asterisks denoted significant differences: ${ }^{*} p \leq 0.05 ;{ }^{* * *} p \leq 0.01$ ).

\subsection{Identification of cis-Acting Elements in the Promoter Region of GASA Genes}

To predict the potential biological functions of the GASA genes, the cis-acting elements of the promoters were analyzed. The cis-acting elements in the promoters of GASA genes related to plant growth and development, hormone response, and stress response were displayed (Figure 9 and Supplementary Figures S4 and S5). The cis-acting regulatory element related to meristem expression, CAT-box, was found in about half of GASA promoters. O2-site, the cis-acting regulatory element involved in zein metabolism regulation, were found in eight PeuGASA promoters, whereas in four PtrGASA promoters. Almost all promoters of GASA genes had the cis-acting element ABRE involved in the abscisic acid responsiveness and the cis-acting element ERE involved in ethylene responsiveness, especially ERE elements were abundant. The cis-acting elements ABRE in the GASA gene promoters of P. trichocarpa were 1.7 times that of P. euphratica. About half of GASA gene promoters had cis-acting elements involved in gibberellin-responsiveness $(52.39 \%$ in $P$. trichocarpa, $42.11 \%$ in P. euphratica). Only one P-box element (gibberellin-responsive element) was found in the GASA gene promoters of P. euphratica (PeuGASA18), while eight P-box elements were found in the seven GASA gene promoters of $P$. trichocarpa. The cis-acting elements involved in mechanical damage responsiveness in the GASA gene promoters of $P$. euphratica were more than those of $P$. trichocarpa, totally accounting for $30 \%$. The cis-acting 
element LTR involved in low-temperature responsiveness and TC-rich repeats involved in defense and stress responsiveness in the GASA gene promoters of $P$. trichocarpa were significantly more than those of P. euphratica. Besides, GASA gene promoters with cis-acting elements involved in stress responsiveness, STRE, accounted for $57.89 \%$ in P. trichocarpa, while $38.10 \%$ in P. euphratica.
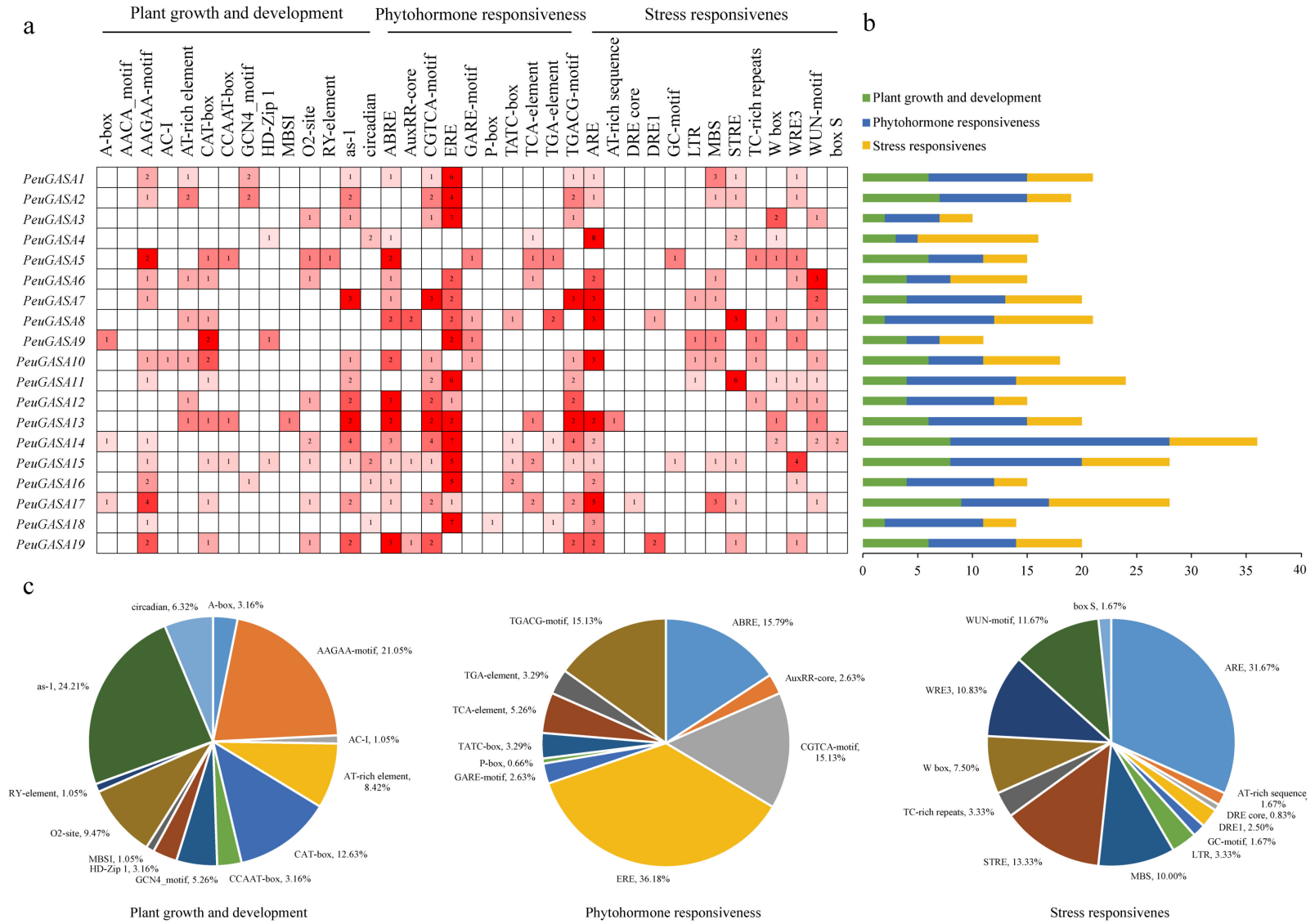

Figure 9. The cis-acting elements of PeuGASA genes. (a) Numbers and gradient red colors indicate the number of cis-acting elements; (b) Color-coded histograms indicate the number of cis-acting elements of genes in each category; (c) Pie charts show the proportion of different cis-acting elements in each category.

\subsection{Analysis of PeuGASA15 Promoter Activity}

Compared with other PeuGASA genes, PeuGASA15 was highly expressed in multiple tissues and its expression responded to hormones and drought stress indicating that PeuGASA15 might be actively involved in various stress regulatory networks, hormone signaling, and metabolic pathways in the GASA gene family. To verify the temporal and spatial expression patterns of PeuGASAs, PeuGASA15 was selected as the representative gene. GUS activity in transgenic Arabidopsis at different growth and development stages was detected by histochemical staining (Figure 10, Supplementary Figure S6). In the initial stage of vegetative growth of pGASA15::GUS transgenic Arabidopsis, strong GUS activity was detected in cotyledons, leaves, hypocotyls, roots, especially in their vascular tissues (Figure 10a-e, Supplementary Figure S6c). GUS activity detected in cotyledons was still strong at the peak of vegetative growth (Figure 10f). Strong GUS activity was detected in several guard cells of leaves (Supplementary Figure S6b). In young leaves unexpanded, GUS activity was mainly detected in leaf tips and vascular bundles. GUS activity was not uniform in roots, and strong GUS activity was detected in the initial and growing parts of lateral roots and the tip parts of taproots (Supplementary Figure S6c-g). During the reproductive growth stage, GUS activity increased with the development of the flower, 
and then gradually decreased. GUS activity was detected in sepals, petals, stigmas and stamens of flowers, especially in their vascular bundles (Figure 10h-m). GUS activity in the parenchyma tissues and vascular bundles of pGASA15::GUS transgenic Arabidopsis flower stems was detected (Supplementary Figure S6h-i). Besides, GUS activity was detected in the funiculus of the dissected silique (Figure 10q). GUS activity of 3-week-old transgenic seedlings was significantly increased in $1 / 2 \mathrm{MS}$ agar medium with $20 \mathrm{mg} / \mathrm{L}$ IAA for $4 \mathrm{~h}$ and decreased in 1/2 MS ager medium with $20 \mu \mathrm{M}$ ABA for $24 \mathrm{~h}$ (Figure 11). The expression patterns of pGASA15::GUS were consistent with the result of qRT-PCR. Mannitol strongly inhibited PeuGASA15 expression in the leaves of transgenic Arabidopsis. $\mathrm{NaCl}$ also had a strong inhibitory effect on PeuGASA15 expression in the unexpanded leaves of pGASA15::GUS transgenic Arabidopsis (Figure 11).

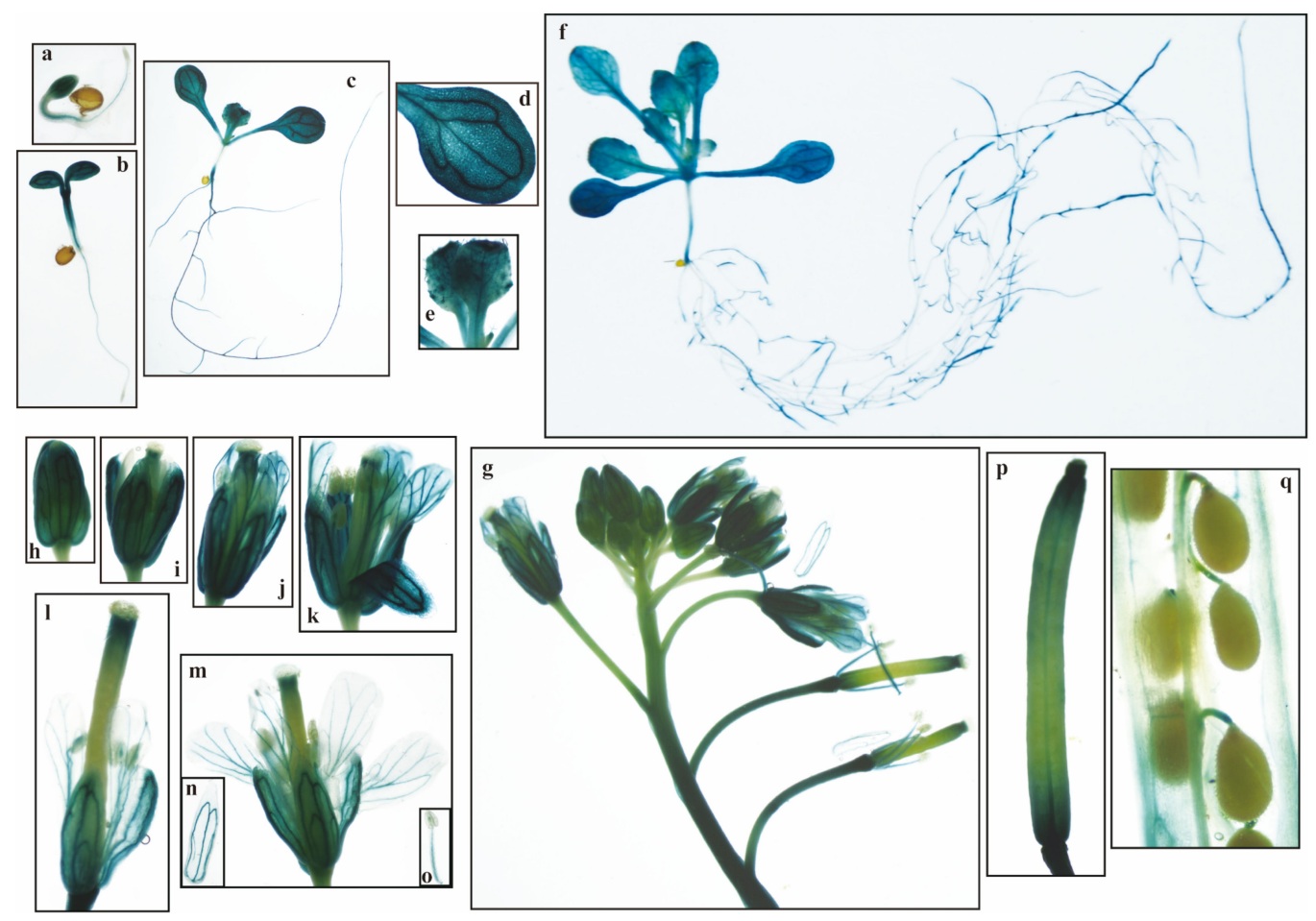

Figure 10. GUS activity of the PeuGASA15 promoter. (a) 3-day-old plant; (b) 4-day-old plant; (c) 7-day-old plant; (d,e) Cotyledons and young leaves of 7-day-old plant; (f) 14d-day-old plant; (g) inflorescence; (h-k) Expanding flowers; (1) Fading flowers; (m) Expanded flower; (n) Petal; (o) Stamen; (p,q) Silique.
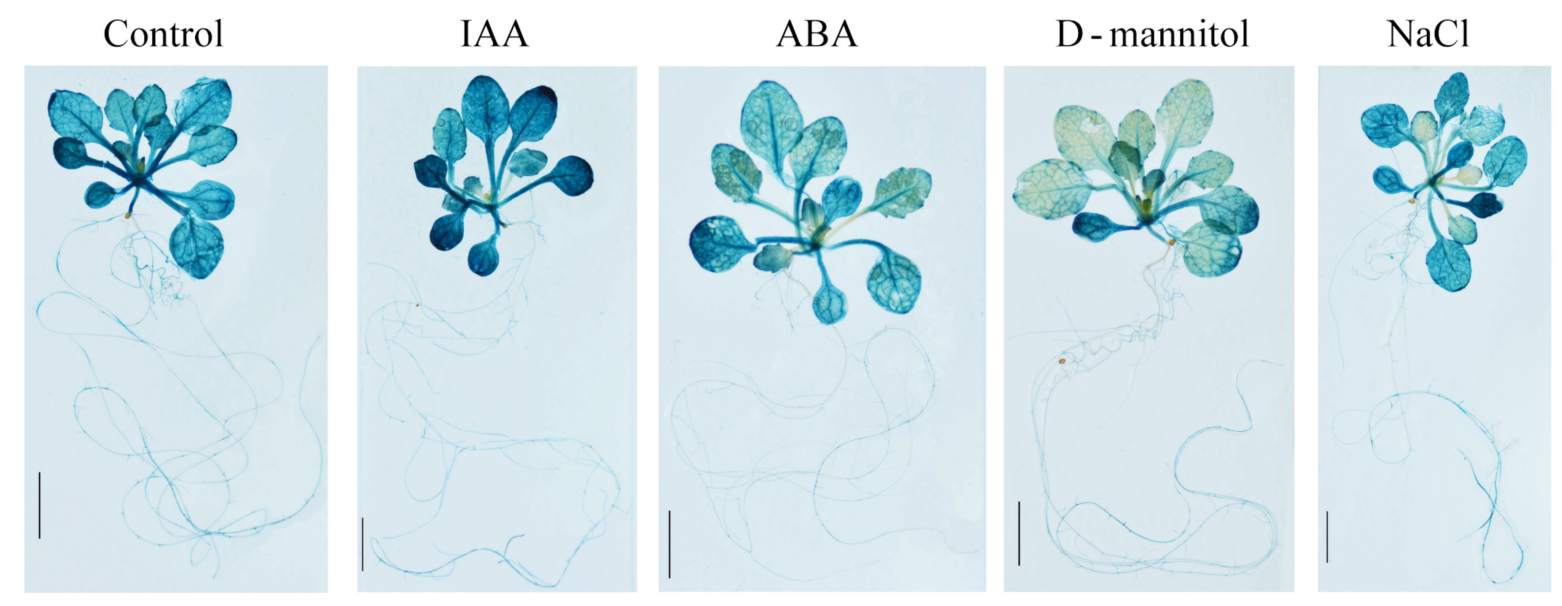

Figure 11. Three-week-old ProGASA15::GUS transgenic Arabidopsis seedlings growing on 1/2 MS AGAR plates were transplanted into medium without or containing $300 \mathrm{mM}$ mannitol, $150 \mathrm{mM} \mathrm{NaCl}, 20 \mu \mathrm{M} \mathrm{ABA}, 20 \mathrm{mg} / \mathrm{L}$ IAA, respectively. 


\section{Discussion}

GASAs, a class of cysteine-rich small peptides, consists of a putative signal peptide at the $\mathrm{N}$-terminal and a conserved GASA domain containing 12 cysteine residues at the C-terminal. GASA proteins play an important role in many aspects of plant growth and development, biotic and abiotic stresses responses, and hormonal signaling [8,13,15,17-19,27,32]. Extensive studies about the biological functions and bioinformatics analysis of GASA genes have been reported in many plants, but little information concerning P. euphratica has been known. In this study, 21 and 19 candidate GASA genes were identified in P. trichocarpa and P. euphratica, respectively, and the bioinformatics and expression patterns of these genes were analyzed. There were candidate GASA genes in P. trichocarpa, but no corresponding homologous genes have been found in $P$. euphratica. This may be because the genome of $P$. euphratica was not as well sequenced as that of $P$. trichocarpa. It is also possible that these extra members formed in P. trichocarpa after two species differentiated, or that these ancestral sequences were lost in P. euphratica [43].

Like potato and apple, the GASA genes were distributed unevenly on several chromosomes in $P$. trichocarpa (Figure 1), while they were distributed on all chromosomes in Arabidopsis [15,44]. The proteins encoded by the GASA genes identified were low molecular weight proteins, which were consistent with the results of Arabidopsis [29]. GASA proteins were mainly predicted to be signal peptides secreted through the classical secretion pathway and localized in extracellular. At present, numerous experiments in vivo have proved that most of the GASA proteins are localized in the cell wall or extracellular region [17,45-48]. GASA genes have not been found in Physcomitrella patens whose whole genome has been sequenced, possibly because GASA genes appeared in vascular plants and originated from pteridophytes [9]. Due to the difference in occurrence and distance between the motifs, these GASA domains were distributed discretely in the phylogenetic tree and mainly divided into three clusters (Figure 4). In subfamily B, there were successive and independent interspecific clades, suggesting that the GASA domains of subfamily B might be relatively ancient and conserved among species (Figure 2). The GASA domains of S. moellendorffii were not found in the subfamily A, suggesting that this subfamily might have evolved late and originated after pteridophytes. SM00035G00570 separated early from subfamily $\mathrm{C}$, indicating that its evolution was completed earlier and might be the origin of this subfamily. Compared with other species, GASAs of poplar and Arabidopsis had relatively higher substitution rates and closer genetic relationships.

Gene replication drives the evolution of genomes and genetic systems and contributes to the diversity of gene structure and function in gene families. WGD and TD are considered to be the two main causes of plant gene family expansion [49]. The number of WGD genes decreased significantly with the prolongation of replication event time, while those of TD genes did not decrease with time. Although the number of derived duplicated genes decreased exponentially, certain WGD gene pairs were still retained in P. trichocarpa and P. euphratica (Table 2). WGD genes in subfamily B degenerated into nonfunctional or disappeared in the long-term evolution which might lead to the deletion of GASA genes in P. euphratica. WGD genes played a dominant role in the evolution of poplar GASA genes in subfamily B. Different from other species with relatively uniform distribution in each subfamily, the GASAs of P. euphratica and P. trichocarpa mostly belong to subfamily A due to the gene expansion. GASA genes in subfamily A retained the conserved WGD genes and evolved new TD genes. TD genes provided variation for adapting to changing environments and tended to evolve subfunctions or new functions involved in plant defense, while WGD genes were relatively conserved [50]. GASA genes in subfamily C neither retained WGD genes nor generated TD genes. The expansion of subfamily $C$ genes might be mainly due to transposition events.

The cis-acting elements predicted in the GASA gene promoters of Populus are roughly identical (Figure 9 and Supplementary Figures S4 and S5). However, there were significant differences in the number of cis-acting elements involved in response to abscisic acid, gibberellin, mechanical damage, low temperature, drought between the promoters of GASA gene families in P. trichocarpa and P. euphratica. The expression patterns of the $P$. 
euphratica GASA gene family were roughly consistent with those of $P$. trichocarpa. After drought treatment, the expression trends of WGD gene pairs, PeuGASA9 and PeuGASA15, PeuGASA6 and PeuGASA13 were consistent respectively. The expression trends of another WGD gene pair, PeuGASA10 and PeuGASA19, were opposite (Figure 8). This tendency was identical with their homologous genes in P. trichocarpa. And there were almost similar expression trends between WGD pair genes after hormonal treatment in P. euphratica mature leaves. Whereas, the expression trends of TD gene pairs were consistent in $P$. trichocarpa which did not share in P. euphratica under drought stress. Partial divergence also appeared in the expression trends of TD gene pairs after hormonal treatment. The long-term adaptation to the complex terrestrial environments might lead to the differences in GASA genes expression between P. euphratica and P. trichocarpa.

GASA1, as a representative gene of GA signal response and transduction, has been mentioned in many reports [51-56]. GASA1 is involved in cell elongation, reproductive growth, leaf senescence, biological and abiotic stress regulatory networks $[34,37,52,53,56,57]$. GASA1 expression also responded to abscisic acid, brassinosteroid and auxin [29,58,59]. AtGASA1, the homolog of PeuGASA15, has been reported to weakly interact with VIT by phage display technology [60]. VIT, also named TETRATRICOPEPTIDE-REPEAT THIOREDOXIN-LIKE 1 (TTL3), plays an important role in brassinosteroid and auxin signaling, and is required for osmotic stress tolerance [60-65]. Consistent with Arabidopsis, IAA induced PeuGASA15 expression and mannitol significantly inhibited PeuGASA15 expression in the leaves of pGASA15: GUS transgenic leaves (Figure 11). GUS gene was highly expressed in various organs of the whole pGASA15: GUS transgenic plant, especially in vascular tissues (Figure 11 and Supplementary Figure S6). Vascular tissues are present in vascular plants, and their main functions are to transport water, inorganic salts, and nutrients and to provide mechanical support for plants [66]. The TTL gene family, like the GASA gene family, is endemic to land plants. VIT also interacted with VH1/BRL2 which plays a role in vascular development [60]. This finding might prove that PeuGASAs are involved in the growth and development of vascular tissues, and provide evidence that the origin of the GASA gene family in vascular plants might be related to their functions.

The expression patterns of genes in different tissues or organs indicate the possible biological functions of these genes [67]. The expression levels of PeuGASA genes in young leaves were relatively high but decreased with leaf maturity, suggesting that PeuGASA genes might be involved in leaf growth and development (Figure 6) [20]. Auxin is mainly distributed in the growth vigorous and young tissues of plants [68,69]. GASA genes were mainly expressed in the young tissues of Populus, and the expression of PeuGASA genes could be induced by auxin, indicating that $G A S A$ genes might play a role in the auxin signal pathway. Gibberellin regulates embryo development, seed germination, stem and root elongation, and flower organ development [70,71]. GASA genes are GA target genes located downstream of the GA signaling pathway [29]. The expression of the GASA gene family was strongly inhibited or induced by gibberellin (Figure 7). It has been reported that the GASA gene family was involved in seed germination, shoot and stem elongation, lateral root formation, flowering and flower development, fruit development, and ripening [15-19,21-25,28]. PeuGASAs were also induced or inhibited by EBR, ABA, SA, and MeJA, indicating that GASAs might be extensively involved in the complex plant hormonal signal transduction network [26]. The cis-acting elements responding to stress hormones and stresses in the promoters of PtrGASA and PeuGASA genes and the expression of PtrGASA and PeuGASA genes was affected by drought, mechanical damage, or other stresses, indicating that PtrGASAs and PeuGASAs play roles in the stress environments. In conclusion, PeuGASAs may be involved in the growth and development, plant hormone responses and signal transduction, and responses to abiotic stresses.

\section{Materials and Methods}

\subsection{Identification of GASA Family Genes in P. trichocarpa and P. euphratica}

The Hidden Markov Model (HMM) profile of the GASA domain (PF02704) from the Pfam database was used as a query and the putative GASA protein sequences were 
identified by the HMMER searching against the P. euphratica genome. These sequences were further confirmed by using Pfam 34.0 database (http:/ / pfam.xfam.org/, accessed on 13 July 2021) and blasted by using Protein BLAST in the NCBI database (https:/ / blast.ncbi. nlm.nih.gov / Blast.cgi, accessed on 15 July 2021). The genomic, transcript, CDS, peptide and, $2000 \mathrm{bp}$ upstream of the translational start site (ATG) promoter region sequences in P. euphratica were extracted from the NCBI database (https:/ / www.ncbi.nlm.nih.gov/, accessed on 2 September 2021). The GASA genes in P. trichocarpa were obtained from the Phytozome 12.0 database (https:/ / phytozome.jgi.doe.gov/pz/portal.html\#!info?alias= Org_Ptrichocarpa, accessed on 18 July 2021) and confirmed by the above method. The PeuGASAs and PtrGASAs were named according to their position on the scaffolds and chromosomes respectively. The PeuGASAs homologous genes in P. trichocarpa were blasted by Protein BLAST and finally confirmed by MEGA 6.0 (Tokyo Metropolitan University, Hachioji, Japan) [72]. The gene position of PtrGASAs and chromosome size were obtained from Phytozome 12.0 and visualized by the TBtools (South China Agricultural University, Guangzhou, China). The cis-acting elements of promoters were predicted using PlantCARE (http:/ / bioinformatics.psb.ugent.be/webtools/plantcare/html/, accessed on 5 September 2021) and classified by reference [73-75]. The results were subsequently visualized by Morpheus (https://software.broadinstitute.org/morpheus/, accessed on 8 September 2021) and Excel (Microsoft, Redmond, WA, USA).

\subsection{Physical and Chemical Properties, Subcellular Localization, Multiple Alignments and Collinearity Analysis}

Physical and chemical parameters of GASAs were computed by ProtParam (https: / / web.expasy.org/protparam/, accessed on 15 August 2021). The subcellular localization sites of GASAs were predicted by WoLF PSORT (https:/ / wolfpsort.hgc.jp/, accessed on 16 August 2021) and TargetP 1.1 Server (http:/ / www.cbs.dtu.dk/services/TargetP-1.1/ index.php, accessed on 17 August 2021). The PeuGASA and PtrGASA protein sequences were multiple aligned by DNAMAN Version 7 , and the multiple sequences alignment was visualized with WebLogo version 2.8.2 (http://weblogo.berkeley.edu/logo.cgi, accessed on 12 August 2021). The gene structure map of GASA genes was drawn by using the gene structure visualization server GSDS2.0 (http:/ /gsds.gao-lab.org/, accessed on 11 September 2021). GASA proteins sequences were submitted to MEME Suite 5.3.3 (https:/ / meme-suite.org/meme/tools/meme, accessed on 12 September 2021) to predict the conserved motifs with default settings for parameters (the maximum number of motifs set to 5). Candidate GASA domains were aligned with the default parameters using MEGA 6.0 [72]. The phylogenetic trees were constructed based on the neighbor-joining method with a bootstrap test of 1000 replicates. The occurrence replication events of GASA genes in P. trichocarpa were analyzed and visualized by MCScanX (University of Georgia, Athens, GA, USA) [76], blast, and TBtools [77]. The above software was also used for the collinearity analysis of GASA genes in Arabidopsis and P. trichocarpa genome.

\subsection{Plant Materials and Sample Collections}

One-year-old Populus euphratica seedlings from Xinjiang Uygur Autonomous Region of China were cultivated in the plastic greenhouse. Each pot contained 3-5 seedlings. The potted seedlings were grown for about 3-4 months under environmentally controlled conditions, and three potted seedlings with the same growth were selected for each treatment. The selected seedlings were treated with $100 \mu \mathrm{M}$ GA3, $200 \mu \mathrm{M} \mathrm{ABA}, 100 \mathrm{mg} / \mathrm{L}$ IAA, $10 \mu \mathrm{M}$ EBR, $100 \mu \mathrm{M} \mathrm{SA}$, and $100 \mu \mathrm{M}$ MeJA respectively. The control group was an aqueous solution containing $0.1 \%$ anhydrous ethanol and $0.02 \%(v / v)$ Triton X-100 [78,79]. Sprayed the whole plant evenly until the leaves are about to drip. Then collected the mature leaves of the seedlings at $0,0.5,1,2,4,8,12$, and $24 \mathrm{~h}$ after treatment. Drought treatment of seedlings was performed according to the method of Tang et al. [37]. All the collected samples were immediately frozen in liquid nitrogen and stored at $-80^{\circ} \mathrm{C}$. 


\subsection{RNA Extraction, cDNA Synthesis, and qRT-PCR Analysis}

The total RNA of samples was extracted using EasySpin Plus Plant RNA Kit (Aidlab Biotech, Beijing, China). The first cDNA strand was synthesized by FastQuant RT Kit (with gDNase, Tiangen, Beijing, China). The specific primers were designed by Primer Premier 6.0 and examined by NCBI Primer-BLAST (https:/ / www.ncbi.nlm.nih.gov/ tools/primerblast/, accessed on 15 July 2021) (Supplementary Table S4). The selection of reference genes of $P$. euphratica was based on the research of Wang et al. [80,81]. Real-time PCR was run with the CFX96 Touch ${ }^{\mathrm{TM}}$ instrument (Bio-Rad, Hercules, CA, USA) to detect the chemical SYBR Green. The established reaction system was as follows: $10 \mu \mathrm{L} 2 \times$ SuperReal Premix Plus (Tiangen), $0.6 \mu \mathrm{L}$ each forward and reverse primers $(10 \mu \mathrm{M}), 1 \mu \mathrm{L}$ diluted cDNA template, and the finally RNase-free $\mathrm{ddH}_{2} \mathrm{O}$ was added until the total amount was $20 \mu \mathrm{L}$. The reaction procedures were: $95^{\circ} \mathrm{C}$ for $15 \mathrm{~min}, 45$ cycles of $10 \mathrm{~s}$ at $95^{\circ} \mathrm{C}$ and $30 \mathrm{~s}$ at $60^{\circ} \mathrm{C}$. The melting curve analysis was performed immediately after the PCR reaction, i.e., fluorescence intensity was measured for each degree increase from $60{ }^{\circ} \mathrm{C}$ to $95^{\circ} \mathrm{C}$. The relative template abundance in each PCR expansion mixture was calculated by the $2^{-\Delta \Delta C T}$ method [82]. Three biological replicates were used for gene expression analysis, and the expressions of the control samples were set to 1 for normalization. SPSS (IBM, Armonk, NY, USA) was used for statistical analysis, and the LSD test was used to calculate the $p$ value. Finally, the results were presented as histograms by GraphPad Prism 8 software (GraphPad, San Diego, CA, USA) or heat maps by Heml software (University of Science and Technology, Wuhan, China).

\subsection{Subcellular Localization of PeuGASA Proteins}

The full-length coding sequences of PeuGASA genes without stop codon were amplified by PCR gene using gene-specific primers (Supplementary Table S5). To express PeuGASA-GFP fusion proteins, the products were cloned between the Super promoter and GFP gene in the pSUPER1300 (+) vector [83]. The confirmed construct fusion vectors were introduced into Agrobacterium tumefaciens strain GV3101 and transformed into Nicotiana benthamiana leaves via Agrobacterium-mediated gene transformation for transient expression [84]. GFP fusion protein GFP signals were detected 2 days after infiltration using Leica fluorescence microscope DM2500 (Leica Microsystems, Wetzlar, Germany).

\subsection{Arabidopsis Culture, Construction and Transformation of Vector, and GUS Activity Assay}

Arabidopsis was planted on 1/2MS medium containing 3\% (w/v) sucrose and $0.6 \%$ $(\mathrm{W} / \mathrm{V})$ ager at $23{ }^{\circ} \mathrm{C}$ with white light fluorescence and photoperiod of $16 / 8 \mathrm{~h}$ (Light/dark). Seven days after seed germination, Arabidopsis was transplanted into vegetative soil and cultured in an incubator to complete the whole growth cycle. A binary vector was constructed using the cloned PeuGASA15 promoter. The fragments were digested with BamHI and EcoRI enzymes and inserted into the binary vector pCAMBIA1391. The vectors were introduced into Agrobacterium tumefaciens strain GV3101 and transformed into Arabidopsis by inflorescence immersion [85]. The transformants were screened on 1/2MS medium supplemented with $25 \mathrm{mg} / \mathrm{L}$ hygromycin. T2 was used for subsequent experiments. For histochemical GUS analysis, the prepared materials were soaked in GUS staining solution and incubated overnight at $37{ }^{\circ} \mathrm{C}$. Then the leaves were decolorized with $70 \%$ ethanol and observed under a Leica microscope and an OLYMPUS SZ61 stereomicroscope (Olympus Corporation, Hachioji, Japan).

Supplementary Materials: The following are available online at https:/ /www.mdpi.com/article/10 $.3390 /$ ijms222212336/s1.

Author Contributions: S.H., Z.J., W.Y. and X.X. conceived the research and designed the experiments. S.H., Z.J. and M.-X.N. performed the experiments. X.Y. and M.H. participated in the experiments. S.H., Z.J., M.-X.N., X.Y., M.H., Y.Z., W.M. and X.W. participated in the plant material culture and preservation. S.H. and Z.J. wrote the manuscript and contributed equally to the study. W.Y., X.X., C.L. 
and H.-L.W. contributed to the review and editing. All authors discussed the results. All authors have read and agreed to the published version of the manuscript.

Funding: This research was funded by the National Natural Science Foundation of China, grant number 31770649, 32071734 and 31900173.

Institutional Review Board Statement: Not applicable.

Informed Consent Statement: Not applicable.

Data Availability Statement: All data supporting the findings of this study are available within the paper and within its Supplementary Materials published online.

Conflicts of Interest: The authors declare no conflict of interest.

\section{References}

1. Marshall, E.; Costa, L.M.; Gutierrez-Marcos, J. Cysteine-rich peptides (CRPs) mediate diverse aspects of cell-cell communication in plant reproduction and development. J. Exp. Bot. 2011, 62, 1677-1686. [CrossRef]

2. Matsubayashi, Y. Posttranslationally Modified Small-Peptide Signals in Plants. Annu. Rev. Plant Biol. 2014, 65, 385-413. [CrossRef] [PubMed]

3. Liu, X.; Zhang, H.; Jiao, H.; Li, L.; Qiao, X.; Fabrice, M.R.; Wu, J.; Zhang, S. Expansion and evolutionary patterns of cysteine-rich peptides in plants. BMC Genom. 2017, 18, 1-14. [CrossRef] [PubMed]

4. Olsson, V.; Joos, L.; Zhu, S.; Gevaert, K.; Butenko, M.A.; De Smet, I. Look Closely, the Beautiful May Be Small: Precursor-Derived Peptides in Plants. Annu. Rev. Plant Biol. 2019, 70, 153-186. [CrossRef] [PubMed]

5. Kereszt, A.; Mergaert, P.; Montiel, J.; Endre, G.; Kondorosi, E. Impact of Plant Peptides on Symbiotic Nodule Development and Functioning. Front. Plant Sci. 2018, 9, 1026. [CrossRef]

6. Uchi, N.; Fukudome, M.; Nozaki, N.; Suzuki, M.; Osuki, K.-I.; Shigenobu, S.; Uchiumi, T. Antimicrobial Activities of Cysteine-rich Peptides Specific to Bacteriocytes of the Pea Aphid Acyrthosiphon pisum. Microbes Environ. 2019, 34, 155-160. [CrossRef] [PubMed]

7. Zhong, S.; Liu, M.; Wang, Z.; Huang, Q.; Hou, S.; Xu, Y.-C.; Ge, Z.; Song, Z.; Huang, J.; Qiu, X.; et al. Cysteine-rich peptides promote interspecific genetic isolation in Arabidopsis. Science 2019, 364, eaau9564. [CrossRef]

8. Wang, L.; Wang, Z.; Xu, Y.; Joo, S.-H.; Kim, S.-K.; Xue, Z.; Xu, Z.; Wang, Z.; Chong, K. OsGSR1is involved in crosstalk between gibberellins and brassinosteroids in rice. Plant J. 2009, 57, 498-510. [CrossRef]

9. Kumar, A.; Singh, A.; Kumar, P.; Sarkar, A.K. Giberellic Acid-Stimulated Transcript Proteins Evolved through Successive Conjugation of Novel Motifs and Their Subfunctionalization. Plant Physiol. 2019, 180, 998-1012. [CrossRef]

10. Shi, L.; Gast, R.T.; Gopalraj, M.; Olszewski, N.E. Characterization of a shoot-specific, GA3- and ABA-Regulated gene from tomato. Plant J. 1992, 2, 153-159. [PubMed]

11. Almasia, N.I.; Bazzini, A.A.; Hopp, H.E.; Vazquez-Rovere, C. Overexpression of snakin-1 gene enhances resistance to Rhizoctonia solani and Erwinia carotovora in transgenic potato plants. Mol. Plant Pathol. 2008, 9, 329-338. [CrossRef]

12. Balaji, V.; Smart, C.D. Over-expression of snakin-2 and extensin-like protein genes restricts pathogen invasiveness and enhances tolerance to Clavibacter michiganensis subsp. michiganensis in transgenic tomato (Solanum lycopersicum). Transgenic Res. 2012, 21, 23-37. [CrossRef]

13. Darqui, F.S.; Radonic, L.M.; Trotz, P.M.; Lopez, N.; Vazquez Rovere, C.; Hopp, H.E.; Lopez Bilbao, M. Potato snakin-1 gene enhances tolerance to Rhizoctonia solani and Sclerotinia sclerotiorum in transgenic lettuce plants. J. Biotechnol. 2018, $283,62-69$. [CrossRef] [PubMed]

14. Rodriguez-Decuadro, S.; Barraco-Vega, M.; Dans, P.D.; Pandolfi, V.; Benko-Iseppon, A.M.; Cecchetto, G. Antimicrobial and structural insights of a new snakin-like peptide isolated from Peltophorum dubium (Fabaceae). Amino Acids 2018, 50, 1245-1259. [CrossRef]

15. Fan, S.; Zhang, D.; Zhang, L.; Gao, C.; Xin, M.; Tahir, M.M.; Li, Y.; Ma, J.; Han, M. Comprehensive analysis of GASA family members in the Malus domestica genome: Identification, characterization, and their expressions in response to apple flower induction. BMC Genom. 2017, 18, 827. [CrossRef] [PubMed]

16. Moyano-Cañete, E.; Bellido, M.L.; García-Caparrós, N.; Medina-Puche, L.; Amil-Ruiz, F.; González-Reyes, J.A.; Caballero, J.L.; Muñoz-Blanco, J.; Blanco-Portales, R. FaGAST2, a Strawberry Ripening-Related Gene, Acts Together with FaGAST1 to Determine Cell Size of the Fruit Receptacle. Plant Cell Physiol. 2013, 54, 218-236. [CrossRef] [PubMed]

17. Zhang, S.; Yang, C.; Peng, J.; Sun, S.; Wang, X. GASA5, a regulator of flowering time and stem growth in Arabidopsis thaliana. Plant Mol. Biol. 2009, 69, 745-759. [CrossRef] [PubMed]

18. Zhong, C.; Xu, H.; Ye, S.; Wang, S.; Li, L.; Zhang, S.; Wang, X. AtGASA6 Serves as an Integrator of Gibberellin-, Abscisic Acid-and Glucose-Signaling during Seed Germination in Arabidopsis. Plant Physiol. 2015, 169, 2288-2303. [CrossRef]

19. Zimmermann, R.; Sakai, H.; Hochholdinger, F. The Gibberellic Acid Stimulated-Like gene family in maize and its role in lateral root development. Plant Physiol. 2010, 152, 356-365. [CrossRef] 
20. Nahirnak, V.; Almasia, N.I.; Fernandez, P.V.; Hopp, H.E.; Estevez, J.M.; Carrari, F.; Vazquez-Rovere, C. Potato snakin-1 gene silencing affects cell division, primary metabolism, and cell wall composition. Plant Physiol. 2012, 158, 252-263. [CrossRef]

21. Han, M.; Jin, X.; Yao, W.; Kong, L.; Huang, G.; Tao, Y.; Li, L.; Wang, X.; Wang, Y. A Mini Zinc-Finger Protein (MIF) from Gerbera hybrida Activates the GASA Protein Family Gene, GEG, to Inhibit Ray Petal Elongation. Front. Plant Sci. 2017, 8, 1649. [CrossRef] [PubMed]

22. Huang, G.; Han, M.; Jian, L.; Chen, Y.; Sun, S.; Wang, X.; Wang, Y. An ETHYLENE INSENSITIVE3-LIKE1 Protein Directly Targets the GEG Promoter and Mediates Ethylene-Induced Ray Petal Elongation in Gerbera hybrida. Front. Plant Sci. 2020, $10,1737$. [CrossRef] [PubMed]

23. Roxrud, I.; Lid, S.E.; Fletcher, J.C.; Schmidt, E.D.; Opsahl-Sorteberg, H.G. GASA4, one of the 14-member Arabidopsis GASA family of small polypeptides, regulates flowering and seed development. Plant Cell Physiol. 2007, 48, 471-483. [CrossRef]

24. Trapalis, M.; Li, S.F.; Parish, R.W. The Arabidopsis GASA10 gene encodes a cell wall protein strongly expressed in developing anthers and seeds. Plant Sci. 2017, 260, 71-79. [CrossRef]

25. Zhang, H.; Yue, M.; Zheng, X.; Gautam, M.; He, S.; Li, L. The Role of Promoter-Associated Histone Acetylation of Haem Oxygenase-1 (HO-1) and Giberellic Acid-Stimulated Like-1 (GSL-1) Genes in Heat-Induced Lateral Root Primordium Inhibition in Maize. Front. Plant Sci. 2018, 9, 1520. [CrossRef] [PubMed]

26. Zhang, S.; Wang, X. One new kind of phytohormonal signaling integrator: Up-and-coming GASA family genes. Plant Signal. Behav. 2017, 12, e1226453. [CrossRef] [PubMed]

27. An, B.; Wang, Q.; Zhang, X.; Zhang, B.; Luo, H.; He, C. Comprehensive transcriptional and functional analyses of HbGASA genes reveal their roles in fungal pathogen resistance in Hevea brasiliensis. Tree Genet. Genomes 2018, 14, 41. [CrossRef]

28. Cheng, X.; Wang, S.; Xu, D.; Liu, X.; Li, X.; Xiao, W.; Cao, J.; Jiang, H.; Min, X.; Wang, J.; et al. Identification and Analysis of the GASR Gene Family in Common Wheat (Triticum aestivum L.) and Characterization of TaGASR34, a Gene Associated With Seed Dormancy and Germination. Front. Genet. 2019, 10, 980. [CrossRef]

29. Zhang, S.; Wang, X. Expression pattern of GASA, downstream genes of DELLA, in Arabidopsis. Sci. Bull. 2008, 53, 3839-3846. [CrossRef]

30. Qiao, K.; Ma, C.; Lv, J.; Zhang, C.; Ma, Q.; Fan, S. Identification, characterization, and expression profiles of the GASA genes in cotton. J. Cotton Res. 2021, 4, 1-16. [CrossRef]

31. Wang, H.; Wei, T.; Wang, X.; Zhang, L.; Yang, M.; Chen, L.; Song, W.; Wang, C.; Chen, C. Transcriptome Analyses from Mutant Salvia miltiorrhiza Reveals Important Roles for SmGASA4 during Plant Development. Int. J. Mol. Sci. 2018, 19, 2088. [CrossRef]

32. Sun, S.; Wang, H.; Yu, H.; Zhong, C.; Zhang, X.; Peng, J.; Wang, X. GASA14 regulates leaf expansion and abiotic stress resistance by modulating reactive oxygen species accumulation. J. Exp. Bot. 2013, 64, 1637-1647. [CrossRef]

33. Iqbal, A.; Wang, T.; Wu, G.; Tang, W.; Zhu, C.; Wang, D.; Li, Y.; Wang, H. Physiological and transcriptome analysis of heteromorphic leaves and hydrophilic roots in response to soil drying in desert Populus euphratica. Sci. Rep. 2017, 7, 1-15. [CrossRef]

34. Li, B.; Qin, Y.; Duan, H.; Yin, W.; Xia, X. Genome-wide characterization of new and drought stress responsive microRNAs in Populus euphratica. J. Exp. Bot. 2011, 62, 3765-3779. [CrossRef] [PubMed]

35. Ottow, E.A.; Polle, A.; Brosché, M.; Kangasjärvi, J.; Dibrov, P.; Zörb, C.; Teichmann, T. Molecular characterization of PeNhaD1: The first member of the NhaD Na+/H+ antiporter family of plant origin. Plant Mol. Biol. 2005, 58, 75-88. [CrossRef] [PubMed]

36. Zhang, Z.; Chen, Y.; Zhang, J.; Ma, X.; Li, Y.; Li, M.; Wang, D.; Kang, M.; Wu, H.; Yang, Y.; et al. Improved genome assembly provides new insights into genome evolution in a desert poplar (Populus euphratica). Mol. Ecol. Resour. 2020, 20, 781-794. [CrossRef] [PubMed]

37. Tang, S.; Liang, H.; Yan, D.; Zhao, Y.; Han, X.; Carlson, J.E.; Xia, X.; Yin, W. Populus euphratica: The transcriptomic response to drought stress. Plant Mol. Biol. 2013, 83, 539-557. [CrossRef] [PubMed]

38. Yan, D.-H.; Fenning, T.; Tang, S.; Xia, X.; Yin, W. Genome-wide transcriptional response of Populus euphratica to long-term drought stress. Plant Sci. 2012, 195, 24-35. [CrossRef] [PubMed]

39. Huang, D.; Wu, W.; Abrams, S.R.; Cutler, A.J. The relationship of drought-related gene expression in Arabidopsis thaliana to hormonal and environmental factors. J. Exp. Bot. 2008, 59, 2991-3007. [CrossRef]

40. Ma, T.; Wang, J.; Zhou, G.; Yue, Z.; Hu, Q.; Chen, Y.; Liu, B.; Qiu, Q.; Wang, Z.; Zhang, J.; et al. Genomic insights into salt adaptation in a desert poplar. Nat. Commun. 2013, 4, 2797. [CrossRef]

41. Yang, Q.; Han, X.M.; Gu, J.K.; Liu, Y.J.; Yang, M.J.; Zeng, Q.Y. Functional and structural profiles of GST gene family from three Populus species reveal the sequence-function decoupling of orthologous genes. New Phytol. 2018, 221, 1060-1073. [CrossRef]

42. Tuskan, G.A.; Difazio, S.; Jansson, S.; Bohlmann, J.; Grigoriev, I.; Hellsten, U.; Putnam, N.; Ralph, S.; Rombauts, S.; Salamov, A.; et al. The genome of black cottonwood, Populus trichocarpa (Torr. \& Gray). Science 2006, 313, 1596-1604.

43. Ma, J.; Lu, J.; Xu, J.; Duan, B.; He, X.; Liu, J. Genome-wide Identification of WRKY Genes in the Desert Poplar Populus euphratica and Adaptive Evolution of the Genes in Response to Salt Stress. Evol. Bioinform. Online 2015, 11 (Suppl. 1), 47-55. [CrossRef] [PubMed]

44. Nahirñak, V.; Rivarola, M.; Gonzalez de Urreta, M.; Paniego, N.; Hopp, H.E.; Almasia, N.I.; Vazquez-Rovere, C. Genome-wide Analysis of the Snakin/GASA Gene Family in Solanum tuberosum cv. Kennebec. Am. J. Potato Res. 2016, 93, 172-188. [CrossRef]

45. Ben-Nissan, G.; Lee, J.-Y.; Borohov, A.; Weiss, D. GIP, a Petunia hybrida GA-induced cysteine-rich protein: A possible role in shoot elongation and transition to flowering. Plant J. 2004, 37, 229-238. [CrossRef] 
46. Furukawa, T.; Sakaguchi, N.; Shimada, H. Two OsGASR genes, rice GAST homologue genes that are abundant in proliferating tissues, show different expression patterns in developing panicles. Genes Genet. Syst 2006, 81, 171-180. [CrossRef] [PubMed]

47. Li, K.-L.; Bai, X.; Li, Y.; Cai, H.; Ji, W.; Tang, L.-L.; Wen, Y.-D.; Zhu, Y.-M. GsGASA1 mediated root growth inhibition in response to chronic cold stress is marked by the accumulation of DELLAs. J. Plant Physiol. 2011, 168, 2153-2160. [CrossRef] [PubMed]

48. Peng, J.; Lai, L.; Wang, X. PRGL: A cell wall proline-rich protein containning GASA domain in Gerbera hybrida. Sci. China Ser. C Life Sci. 2008, 51, 520-525. [CrossRef]

49. Cannon, S.B.; Mitra, A.; Baumgarten, A.; Young, N.D.; May, G. The roles of segmental and tandem gene duplication in the evolution of large gene families in Arabidopsis thaliana. BMC Plant Biol. 2004, 4, 10. [CrossRef]

50. Qiao, X.; Li, Q.; Yin, H.; Qi, K.; Li, L.; Wang, R.; Zhang, S.; Paterson, A.H. Gene duplication and evolution in recurring polyploidization-diploidization cycles in plants. Genome Biol. 2019, 20, 1-23. [CrossRef]

51. Alonso-Peral, M.M.; Li, J.; Li, Y.; Allen, R.S.; Schnippenkoetter, W.; Ohms, S.; White, R.G.; Millar, A.A. The microRNA159regulated GAMYB-like genes inhibit growth and promote programmed cell death in Arabidopsis. Plant Physiol. 2010, 154, 757-771. [CrossRef] [PubMed]

52. Zhang, S.; Shi, Q.; Albrecht, U.; Shatters, R.G., Jr.; Stange, R.; McCollum, G.; Zhang, S.; Fan, C.; Stover, E. Comparative transcriptome analysis during early fruit development between three seedy citrus genotypes and their seedless mutants. Hortic. Res. 2017, 4, 17041. [CrossRef]

53. Gao, X.; Wang, L.; Zhang, H.; Zhu, B.; Lv, G.; Xiao, J. Transcriptome analysis and identification of genes associated with floral transition and fruit development in rabbiteye blueberry (Vaccinium ashei). PLoS ONE 2021, 16, e0259119. [CrossRef] [PubMed]

54. Chen, X.; Guo, W.; Liu, B.; Zhang, Y.; Song, X.; Cheng, Y.; Zhang, L.; Zhang, T. Molecular mechanisms of fiber differential development between G. barbadense and G. hirsutum revealed by genetical genomics. PLoS ONE 2012, 7, e30056. [CrossRef] [PubMed]

55. Lin, M.; Pang, C.; Fan, S.; Song, M.; Wei, H.; Yu, S. Global analysis of the Gossypium hirsutum L. Transcriptome during leaf senescence by RNA-Seq. BMC Plant Biol. 2015, 15, 43. [CrossRef]

56. Tobar, M.; Fiore, N.; Perez-Donoso, A.G.; Leon, R.; Rosales, I.M.; Gambardella, M. Divergent molecular and growth responses of young "Cabernet Sauvignon" (Vitis vinifera) plants to simple and mixed infections with Grapevine rupestris stem pittingassociated virus. Hortic. Res. 2020, 7, 1-14. [CrossRef]

57. Dias, P.M.; Brunel-Muguet, S.; Durr, C.; Huguet, T.; Demilly, D.; Wagner, M.H.; Teulat-Merah, B. QTL analysis of seed germination and pre-emergence growth at extreme temperatures in Medicago truncatula. Theor. Appl. Genet. 2011, 122, 429-444. [CrossRef]

58. Bouquin, T.; Meier, C.; Foster, R.; Nielsen, M.E.; Mundy, J. Control of Specific Gene Expression by Gibberellin and Brassinosteroid. Plant Physiol. 2001, 127, 450-458. [CrossRef]

59. Paponov, I.A.; Paponov, M.; Teale, W.; Menges, M.; Chakrabortee, S.; Murray, J.A.; Palme, K. Comprehensive transcriptome analysis of auxin responses in Arabidopsis. Mol. Plant 2008, 1, 321-337. [CrossRef]

60. Ceserani, T.; Trofka, A.; Gandotra, N.; Nelson, T. VH1/BRL2 receptor-like kinase interacts with vascular-specific adaptor proteins VIT and VIK to influence leaf venation. Plant J. 2009, 57, 1000-1014. [CrossRef]

61. Lakhssassi, N.; Doblas, V.G.; Rosado, A.; del Valle, A.E.; Pose, D.; Jimenez, A.J.; Castillo, A.G.; Valpuesta, V.; Borsani, O.; Botella, M.A. The Arabidopsis tetratricopeptide thioredoxin-like gene family is required for osmotic stress tolerance and male sporogenesis. Plant Physiol. 2012, 158, 1252-1266. [CrossRef]

62. Amorim-Silva, V.; Garcia-Moreno, A.; Castillo, A.G.; Lakhssassi, N.; Esteban Del Valle, A.; Perez-Sancho, J.; Li, Y.; Pose, D.; Perez-Rodriguez, J.; Lin, J.; et al. TTL Proteins Scaffold Brassinosteroid Signaling Components at the Plasma Membrane to Optimize Signal Transduction in Arabidopsis. Plant Cell 2019, 31, 1807-1828. [CrossRef]

63. Rosado, A.; Schapire, A.L.; Bressan, R.A.; Harfouche, A.L.; Hasegawa, P.M.; Valpuesta, V.; Botella, M.A. The Arabidopsis tetratricopeptide repeat-containing protein TTL1 is required for osmotic stress responses and abscisic acid sensitivity. Plant Physiol. 2006, 142, 1113-1126. [CrossRef]

64. Cuadrado-Pedetti, M.B.; Rauschert, I.; Sainz, M.M.; Amorim-Silva, V.; Botella, M.A.; Borsani, O.; Sotelo-Silveira, M. The Arabidopsis TETRATRICOPEPTIDE THIOREDOXIN-LIKE 1 Gene Is Involved in Anisotropic Root Growth during Osmotic Stress Adaptation. Genes 2021, 12, 236. [CrossRef] [PubMed]

65. Imtiaz, M.; Yang, Y.; Liu, R.; Xu, Y.; Khan, M.A.; Wei, Q.; Gao, J.; Hong, B. Identification and functional characterization of the BBX24 promoter and gene from chrysanthemum in Arabidopsis. Plant Mol. Biol. 2015, 89, 1-19. [CrossRef] [PubMed]

66. Lucas, W.J.; Groover, A.; Lichtenberger, R.; Furuta, K.; Yadav, S.R.; Helariutta, Y.; He, X.Q.; Fukuda, H.; Kang, J.; Brady, S.M.; et al. The plant vascular system: Evolution, development and functions. J. Integr. Plant Biol. 2013, 55, 294-388. [CrossRef] [PubMed]

67. Ray, S.; Agarwal, P.; Arora, R.; Kapoor, S.; Tyagi, A.K. Expression analysis of calcium-dependent protein kinase gene family during reproductive development and abiotic stress conditions in rice (Oryza sativa L. ssp. indica). Mol. Genet. Genom. 2007, 278, 493-505. [CrossRef]

68. Ljung, K.; Hull, A.K.; Celenza, J.; Yamada, M.; Estelle, M.; Normanly, J.; Sandberg, G. Sites and regulation of auxin biosynthesis in Arabidopsis roots. Plant Cell 2005, 17, 1090-1104. [CrossRef] [PubMed]

69. Powers, S.K.; Strader, L.C. Regulation of auxin transcriptional responses. Dev. Dyn. 2020, 249, 483-495. [CrossRef] [PubMed]

70. Yamaguchi, N.; Winter, C.M.; Wu, M.F.; Kanno, Y.; Yamaguchi, A.; Seo, M.; Wagner, D. Gibberellin acts positively then negatively to control onset of flower formation in Arabidopsis. Science 2014, 344, 638-641. [CrossRef] 
71. Rizza, A.; Jones, A.M. The makings of a gradient: Spatiotemporal distribution of gibberellins in plant development. Curr. Opin. Plant Biol. 2019, 47, 9-15. [CrossRef]

72. Tamura, K.; Stecher, G.; Peterson, D.; Filipski, A.; Kumar, S. MEGA6: Molecular Evolutionary Genetics Analysis version 6.0. Mol. Biol. Evol. 2013, 30, 2725-2729. [CrossRef]

73. Yu, Z.; Zhao, C.; Zhang, G.; Teixeira da Silva, J.A.; Duan, J. Genome-Wide Identification and Expression Profile of TPS Gene Family in Dendrobium officinale and the Role of DoTPS10 in Linalool Biosynthesis. Int. J. Mol. Sci. 2020, 21, 5419. [CrossRef] [PubMed]

74. Huang, W.; Hu, B.; Liu, J.; Zhou, Y.; Liu, S. Identification and Characterization of Tonoplast Sugar Transporter (TST) Gene Family in Cucumber. Hortic. Plant J. 2020, 6, 145-157. [CrossRef]

75. Chao, J.; Huang, Z.; Yang, S.; Deng, X.; Tian, W. Genome-wide identification and expression analysis of the phosphatase 2A family in rubber tree (Hevea brasiliensis). PLoS ONE 2020, 15, e228219. [CrossRef] [PubMed]

76. Wang, Y.; Tang, H.; Debarry, J.D.; Tan, X.; Li, J.; Wang, X.; Lee, T.H.; Jin, H.; Marler, B.; Guo, H.; et al. MCScanX: A toolkit for detection and evolutionary analysis of gene synteny and collinearity. Nucleic Acids Res. 2012, 40, e49. [CrossRef] [PubMed]

77. Chen, C.; Chen, H.; Zhang, Y.; Thomas, H.R.; Frank, M.H.; He, Y.; Xia, R. TBtools: An Integrative Toolkit Developed for Interactive Analyses of Big Biological Data. Mol. Plant 2020, 13, 1194-1202. [CrossRef]

78. Wang, D.; Gao, Z.; Du, P.; Xiao, W.; Tan, Q.; Chen, X.; Li, L.; Gao, D. Expression of ABA Metabolism-Related Genes Suggests Similarities and Differences Between Seed Dormancy and Bud Dormancy of Peach (Prunus persica). Front Plant Sci. 2015, 6, 1248. [CrossRef]

79. Zhang, H.; Hong, Y.; Huang, L.; Liu, S.; Tian, L.; Dai, Y.; Cao, Z.; Huang, L.; Li, D.; Song, F. Virus-Induced Gene Silencing-Based Functional Analyses Revealed the Involvement of Several Putative Trehalose-6-Phosphate Synthase/Phosphatase Genes in Disease Resistance against Botrytis cinerea and Pseudomonas syringae pv. tomato DC3000 in Tomato. Front Plant Sci. 2016, 7, 1176. [CrossRef] [PubMed]

80. Wang, H.L.; Li, L.; Tang, S.; Yuan, C.; Tian, Q.; Su, Y.; Li, H.G.; Zhao, L.; Yin, W.; Zhao, R.; et al. Evaluation of Appropriate Reference Genes for Reverse Transcription-Quantitative PCR Studies in Different Tissues of a Desert Poplar via Comparision of Different Algorithms. Int. J. Mol. Sci. 2015, 16, 20468-20491. [CrossRef]

81. Wang, H.L.; Zhang, Y.; Wang, T.; Yang, Q.; Yang, Y.; Li, Z.; Li, B.; Wen, X.; Li, W.; Yin, W.; et al. An alternative splicing variant of PtRD26 delays leaf senescence by regulating multiple NAC transcription factors in Populus. Plant Cell 2021, 33, 1594-1614. [CrossRef]

82. Livak, K.J.; Schmittgen, T.D. Analysis of relative gene expression data using real-time quantitative PCR and the 2(-Delta Delta C(T)) Method. Methods 2001, 25, 402-408. [CrossRef] [PubMed]

83. Zhao, R.; Sun, H.L.; Mei, C.; Wang, X.J.; Yan, L.; Liu, R.; Zhang, X.F.; Wang, X.F.; Zhang, D.P. The Arabidopsis Ca(2+) -dependent protein kinase CPK12 negatively regulates abscisic acid signaling in seed germination and post-germination growth. New Phytol. 2011, 192, 61-73. [CrossRef] [PubMed]

84. Li, X. Infiltration of Nicotiana benthamiana Protocol for Transient Expression via Agrobacterium. Bio-Protocol 2011, 1, e95. [CrossRef]

85. Clough, S.J.; Bent, A.F. Floral dip: A simplified method for Agrobacterium-mediated transformation of Arabidopsis thaliana. Plant J. 1998, 16, 735-743. [CrossRef] 